\title{
Polymer Principles of Protein Calorimetric Two-State Cooperativity
}

\author{
Hüseyin KAYA and Hue Sun CHAN \\ Department of Biochemistry, and \\ Department of Medical Genetics $\&$ Microbiology \\ Faculty of Medicine, University of Toronto \\ Toronto, Ontario M5S 1A8, Canada
}

\begin{abstract}
The experimental calorimetric two-state criterion requires the van't Hoff enthalpy $\Delta H_{\mathrm{vH}}$ around the folding/unfolding transition midpoint to be equal or very close to the calorimetric enthalpy $\Delta H_{\text {cal }}$ of the entire transition. We use an analytical model with experimental parameters from chymotrypsin inhibitor 2 to elucidate the relationship among several different van't Hoff enthalpies used in calorimetric analyses. Under reasonable assumptions, the implications of these $\Delta H_{\mathrm{vH}}$ 's being approximately equal to $\Delta H_{\text {cal }}$ are equivalent: Enthalpic variations among denatured conformations in real proteins are much narrower than some previous lattice-model estimates, suggesting that the energy landscape theory "folding to glass transition temperature ratio" $T_{\mathrm{f}} / T_{\mathrm{g}}$ may exceed 6.0 for real calorimetrically two-state proteins. Several popular three-dimensional lattice protein models, with different numbers of residue types in their alphabets, are found to fall short of the high experimental standard for being calorimetrically two-state. Some models postulate a multiple-conformation native state with substantial pre-denaturational energetic fluctuations well below the unfolding transition temperature and/or predict a significant post-denaturational continuous conformational expansion of the denatured ensemble at temperatures well above the transition point. These scenarios either disagree with experiments on protein size and dynamics, or are inconsistent with conventional interpretation of calorimetric data. However, when empirical linear baseline subtractions are employed, the resulting $\Delta H_{\mathrm{vH}} / \Delta H_{\mathrm{cal}}$ 's for some models can be increased to values closer to unity; and baseline subtractions are found to correspond roughly to an operational definition of native-state conformational diversity. These results necessitate a re-assessment of theoretical models and experimental interpretations.
\end{abstract}

Key words: calorimetry; van't Hoff enthalpy; lattice models; radius of gyration;

baseline subtraction; native state definition

\section{Introduction}

In recent years, protein folding has been investigated extensively by statistical mechanical modeling (see reviews in Refs. 1-14, Refs. 15-23, and references therein). The relevance of these models to the basic understanding of microscopic energetics is premised on the tenet that macroscopic properties of a system are consequences of the properties of its microscopic constituent parts. It follows that insight and rationalization can be gained by constructing models and testing whether the presumed microscopic interactions are effective in reproducing experimental macroscopic behaviors. ${ }^{24}$ High-resolution force-field potentials have been used to study protein folding ${ }^{25}$ and unfolding. ${ }^{26-28}$ Obviously, atomistic models are indispensable for structural details. But at present it is not computationally feasible to use them to model thermodynamics and kinetics at millisecond or longer time scales. Also, it remains an open question whether empirical force fields would ultimately be adequate for predicting dynamics over long simulations. ${ }^{29}$ Currently, a significant fraction of thermodynamics and kinetics data of proteins can only be addressed by complementary approaches, mainly via polymer models with highly simplified representations of the geometry and interactions of the polypeptide chain. ${ }^{1-4,15,30}$ Aside from their computational tractability, it is hoped that these simplified models may lead to the development of novel, (as-yet-undiscovered ${ }^{31}$ ) concepts. Such "mesoscopic" organizing principles ${ }^{31}$ may be needed to bridge our understanding over gaps of many orders of magnitude in time and length scales separating the fundamental constituent atomic processes and the global features of a bio-macromolecule.

\section{Simple self-contained polymer models can be used to explore microscopic energetics of proteins.}

How do simple polymer protein models contribute to our physical understanding of proteins? Typically, the ingredients of such a model are (i) a conformational space that accounts for chain connectivity and excluded volume, and is sufficiently simple to be enumerated exhaustively ${ }^{4,32}$ or sampled extensively, ${ }^{3,7,11}$ and (ii) a set of rules (a potential function) that describes the "microscopic" interactions among the constituent parts of the chain. The most important feature of such a model is the conceptual clarity it offers because it is selfcontained. This means that all properties and predictions of the model are derived solely from the postulated elementary microscopic ingredients. In particular, conformational ensembles are determined by applying the 
model potential function (ii) to ascertain the energetic favorability of every conformation in the model conformational space (i). Most recent lattice protein models belong to this category. However, some protein models are not self-contained in this sense. In some thermodynamic treatments ${ }^{33-36}$, for example, the unfolded or denatured state of a protein is postulated to contain only random-coil-like conformations, but with no specification as to what microscopic interactions are responsible for such a remarkable property in discriminating against compact nonnative conformations. (See discussions in Ref. 23.) As such, non-self-contained models involve either unspecified or unjustified mechanisms that are not explicitly considered as parts of their microscopic potential functions. Therefore, their explanatory power is limited because they cannot make a full logical connection between the macroscopic properties they predict and the microscopic interactions they explicitly consider, though they can provide important insight and be very useful in other respects.

Self-contained simple polymer models of proteins help frame our discourse in terms of basic physical interactions. They sharpen our focus on whether certain global properties can or cannot arise from the microscopic interactions presumed by a model. In these models, however, the necessity to simplify implies that one has to rely to a degree on intuitive judgement in the design of appropriate model representations to capture polypeptide properties. In principle, many simple models can give similar results. A successful predictions can therefore be fortuitous. It follows that the ability to reproduce a protein property is necessary but not sufficient for the validity of the presumed microscopic features of a model. On the other hand, if properties of a model are in disagreement with experimental data, it is a clear indication of deficiencies. Since simple models appear to enjoy a high degree of latitude in their design, it might be expected that reproducing general, "generic" 37 properties of proteins would be straightforward. This is not the case. To the contrary, using simple models with physically plausible interactions to reproduce several thermodynamic ${ }^{23,38}$ and kinetic ${ }^{19,39}$ properties of proteins has been shown to be not trivial and requires in-depth analyses. This may be a blessing in disguise, because it means that a lot can be learnt about microscopic protein energetics from generic protein properties by using the latter as restrictive experimental constraints on models, to provide insight into what form of microscopic interactions are more likely to be proteinlike.

The calorimetric criterion for thermodynamic two-state cooperativity requires a narrow denatured-state enthalpy distribution.

One generic protein property that apparently has not been fully appreciated by modelers is the calori- metric two-state behavior of many small single-domain proteins, ${ }^{40,41}$ which requires that the van't Hoff enthalpy $\Delta H_{\mathrm{vH}}$ around the folding/unfolding transition midpoint to be equal or very close to the calorimetric enthalpy $\Delta H_{\text {cal }}$ of the entire transition. Thermodynamic properties of several simple polymer models have recently been compared with this experimental criterion for two-state cooperativity. ${ }^{22,23}$ One of us $^{23}$ argued that, under reasonable assumptions, the calorimetric two-state condition requires the average enthalpy difference between the denatured and native ensembles around the heat denaturation midpoint not to further increase appreciably as the temperature is raised to complete the unfolding process. From analyses of analytic as well as two-dimensional lattice models, this is found to imply that the enthalpy distribution among the denatured ensemble of conformations has to be narrow in comparison with the average enthalpy difference between the native state and the denatured state. ${ }^{23}$ In the present study, we provide further support for this view by determining systematically the effects of using several slightly different common definitions of van't Hoff enthalpy for the calorimetric two-state criterion.

A number of two-dimensional lattice protein models have been evaluated against the calorimetric criterion. ${ }^{23}$ Interestingly and unexpectedly, both a G⿰ $\overline{\mathrm{o}}^{15,19}$ and a Gō-like HP+ (Ref. 19) model are found to be far away from being calorimetrically two-state. Apparently, insofar as the underlying chain model is highly flexible, even for these models with native-specific pairwise additive contact interactions (these interaction schemes are sometimes referred to as being "nearly maximally unfrustrated" ${ }^{2,43}$ ), the denatured enthalpy distributions in these two-dimensional models are still too board to satisfy the calorimetric two-state standard. Based on these results, it has been suggested that a cooperative interplay between local and nonlocal interactions in proteins may be necessary to give rise to calorimetrically two-state behaviors. ${ }^{23}$ In the present work, we evaluate six three-dimensional lattice protein models. These include two- ${ }^{44}$ and three-letter ${ }^{45}$ models, a Gō model, ${ }^{46}$ a "solvation" model ${ }^{47}$ and 20-letter models with ${ }^{48}$ and without ${ }^{49}$ sidechains. Their thermodynamics are checked against the calorimetric criterion. We also evaluate the physical pictures of native and denatured states offered by some of these models in light of other experimental measurements on protein folding/denaturation transitions.

\section{Results and Discussion}

\section{Overview of an analytical treatment.}

To provide a basic theoretical underpinning, we first re-examine several definitions of van't Hoff enthalpy ( $\Delta H_{\mathrm{vH}}$ 's) in the protein folding literature, and the con- 
sequences of using different $\Delta H_{\mathrm{vH}}$ 's in the calorimetric two-state criterion $\Delta H_{\mathrm{vH}} / \Delta H_{\text {cal }} \approx 1$. The main result of this section, to be demonstrated below, is that under reasonable, minimal assumptions regarding protein conformational properties, calorimetric two-state criteria using several commonly employed $\Delta H_{\mathrm{vH}}$ 's imply essentially equivalent requirements on a protein's density of states. ${ }^{23}$ We approach this by comparing the $\Delta H_{\mathrm{vH}} / \Delta H_{\text {cal }}$ values using different $\Delta H_{\mathrm{vH}}$ 's computed for a series of analytical models with a wide range of thermodynamic cooperativities.

We begin by recalling a few basic relations. As discussed in detail previously, ${ }^{23}$ the main thermodynamic quantities of interest for the issues at hand are the excess enthalpy and heat capacity. Experimentally, raw calorimetric data consists of heat capacity scans over a range of temperatures, from which an excess enthalpy

$$
\langle\Delta H(T)\rangle=\langle H(T)\rangle-H_{\mathrm{N}}
$$

as a function of absolute temperature $T$ can be obtained by standard baseline subtraction and numerical integration techniques. ${ }^{41}$ Here $H$ is the enthalpy of the entire "excess" system, ${ }^{23,41} H_{\mathrm{N}}$ is the enthalpy of the native state, and $\langle\ldots\rangle$ denotes Boltzmann averaging. In general, the native enthalpy $H_{\mathrm{N}}$ should be replaced by a Boltzmann average $\left\langle H_{\mathrm{N}}(T)\right\rangle$ over conformational variations in the native state. (See discussions below on 20-letter models with and without sidechains.) Here we adopt as a working assumption that the native state become effectively a single conformation with a single temperature-independent enthalpy value after proper baseline subtractions. ${ }^{23}$ The calorimetric enthalpy $\Delta H_{\text {cal }}=\left\langle\Delta H\left(T_{1}\right)\right\rangle$ at a sufficiently high temperature $T_{1}$ at which the heat denaturation process is completed ( $T_{1}$ may be formally taken to be $\infty$ in model considerations). ${ }^{23}$ The expression for the excess heat capacity function

$$
C_{P}=\frac{\partial\langle\Delta H(T)\rangle}{\partial T}=\frac{\left\langle H^{2}(T)\right\rangle-\langle H(T)\rangle^{2}}{k_{B} T^{2}},
$$

follows from standard statistical mechanics, ${ }^{23}$ where $k_{B}$ is Boltzmann's constant. Equation (2) corresponds to $\Delta C_{P}$ in the calorimetric literature $\left(\Delta C_{P, \text { tr }}\right.$ in Ref. 41$)$. We drop the symbol $\Delta$ here for the excess heat capacity as in Ref. 23 to simplify notation.

Several different definitions of $\Delta H_{\mathrm{vH}}$ have been put forth in the protein calorimetric literature. ${ }^{22,23,40,41,50,51}$ In general, their values can be very different. This raises the possibility of complications in comparison between theory and experiment. In Ref. 23, one of us noted that while different $\Delta H_{\mathrm{vH}}$ 's may be different when the transition is far from being calorimetrically two state - i.e., two-state as defined by the condition $\Delta H_{\mathrm{vH}} / \Delta H_{\text {cal }} \approx 1$ using any one of the $\Delta H_{\mathrm{vH}}$ 's, a semi-quantitative argument can infer that for proteins which can be fully denatured by heat, $\Delta H_{\mathrm{vH}} \approx \Delta H_{\text {cal }}$ for one $\Delta H_{\mathrm{vH}}$ would imply that the same approximate equality also holds for other $\Delta H_{\mathrm{vH}}$ 's. Here we substantiate this inference by quantitatively analyzing a class of models for protein densities of states.

\section{Definitions of protein folding van't Hoff en- thalpies.}

In general, a temperature-dependent van't Hoff enthalpy is given by

$$
\Delta H_{\mathrm{vH}}(T)=k_{B} T^{2} \frac{d \ln K^{\mathrm{eff}}}{d T}=k_{B} T^{2} \frac{1}{\theta(1-\theta)} \frac{d \theta}{d T},
$$

where $K^{\text {eff }}$ is the apparent ${ }^{52,53}$ or effective ${ }^{22,51}$ equilibrium constant of the system, and $\theta=\theta(T)$ is a two-state progress parameter for tracking the transition process; $K^{\text {eff }}=\theta /(1-\theta)$ and $\theta$ takes values from zero (at low temperatures in the present cases) to unity (at high temperatures). For heat denaturation of proteins, $\theta=0$ and $\theta=1$ correspond respectively to the completely native (fully folded) and fully denatured (unfolded) states. ${ }^{1}$ Therefore, at the midpoint temperature $T_{\text {midpoint }}$ of the parameter $\theta$, i.e., when $\theta\left(T=T_{\text {midpoint }}\right)=1 / 2$,

$$
\Delta H_{\mathrm{vH}}=\left.4 k_{B} T_{\text {midpoint }}^{2} \frac{d \theta}{d T}\right|_{T=T_{\text {midpoint }}} .
$$

As in Ref. 23 and is customary in the calorimetric literature, $\Delta H_{\mathrm{vH}}$ is understood to be evaluated at a certain midpoint temperature when its $T$ dependence is not shown explicity.

It follows that different choices of $\theta$ would result in different van't Hoff enthalpies and different midpoint temperatures. The theoretical population-based $\Delta H_{\mathrm{vH}}$ in Ref. 23 corresponds to $\theta=[\mathrm{D}]$ - the denatured fraction of the total population, and a midpoint temperature $T_{1 / 2}$ at which one half of the chain population is denatured. Here we use $\kappa_{0}$ to denote the $\Delta H_{\mathrm{vH}} / \Delta H_{\text {cal }}$ ratio of this population-based van't Hoff enthalpy to the calorimetric enthalpy. Experimentally, the heat absorbed by the system is often used to quantitate the degree of progress of the transition process under a two-state assumption by setting $\theta=\langle\Delta H\rangle / \Delta H_{\text {cal }}$, with a corresponding midpoint temperature $T_{d}$ at which one half of the total calorimetric heat $\left(\Delta H_{\text {cal }} / 2\right)$ has been absorbed (Ref. 51). This

\footnotetext{
${ }^{1} \theta$ is equivalent to Lumry et al.'s ${ }^{52}\left(\left[\langle\alpha\rangle(T)-\langle\alpha\rangle_{\mathrm{A}}(T)\right] /\right.$ $\left[\langle\alpha\rangle_{\mathrm{B}}(T)-\langle\alpha\rangle_{\mathrm{A}}(T)\right]$, where $\alpha$ is an observable [their Eq. (4)].
} 
leads to a van't Hoff enthalpy which is proportional to the excess specific heat at $T_{d}$ (see below).

On the other hand, a "square-root" van't Hoff enthalpy formula has also been used by Privalov and coworkers ${ }^{40,50}$ to analyze experimental data. It takes the form

$$
\Delta H_{\mathrm{vH}}=2 T_{\text {midpoint }} \sqrt{k_{B} C_{P}\left(T_{\text {midpoint }}\right)} .
$$

Apparently, this corresponds to setting $\theta(T)=$ $\langle\Delta H(T)\rangle / \Delta H_{\mathrm{vH}}$, and assuming that it is a valid progress parameter. Equation (5) is used in conjunction with either the peak temperature $T_{\max }$ of $C_{P}$ (Ref. 40) or $T_{d}$ (Ref. 50) as midpoint temperatures at which $\theta=1 / 2$ is presumably a good approximation (see also Ref. 23). To ascertain the effects of different $\Delta H_{\mathrm{vH}}$ 's on the calorimetric criterion, we compare the population-based $\kappa_{0}$ defined above with the following possible van't Hoff to calorimetric enthalpy ratios using different midpoint temperatures for the square-root formula ${ }^{23,40,50}$ :

$$
\begin{aligned}
\kappa_{1} & =2 T_{1 / 2} \sqrt{k_{B} C_{P}\left(T_{1 / 2}\right)} / \Delta H_{\mathrm{cal}}, \\
\kappa_{2} & =2 T_{\max } \sqrt{k_{B} C_{P}\left(T_{\max }\right)} / \Delta H_{\mathrm{cal}}, \\
\kappa_{3} & =2 T_{d} \sqrt{k_{B} C_{P}\left(T_{d}\right)} / \Delta H_{\mathrm{cal}} .
\end{aligned}
$$

Finally, it is not difficult to see that the van't Hoff to calorimetric enthalpy ratio for $\theta=\langle\Delta H\rangle / \Delta H_{\text {cal }}$ above is given ${ }^{51}$ by $\left(\kappa_{3}\right)^{2}$. So we also consider $\left(\kappa_{1}\right)^{2}\left(\kappa_{2}\right)^{2}$ and $\left(\kappa_{3}\right)^{2}$ as possible van't Hoff to calorimetric enthalpy ratios. The definitions and usage of these quantities are summarized in Table I.

\section{Despite their different definitions, several van't Hoff enthalpies give essentially the same calori- metric two-state criterion.}

We now compute these different van't Hoff to calorimetric enthalpy ratios for a class of models that intuitively capture the most basic features of protein energetics, which are an essentially unique native state as the lowest (ground) enthalpic state of the system, and a huge number of unfolded (denatured) conformations with higher enthalpies. For this purpose, we use simple random-energy-like models with Gaussian enthalpy distributions for the denatured states. Their (continuum) densities of states $\mathrm{g}(H)$ are given by ${ }^{23}$

$$
\mathrm{g}(H)=\delta(H)+\theta(H) \frac{\mathrm{g}_{\mathrm{D}}}{\sqrt{2 \pi} \sigma_{H}} \mathrm{e}^{-\left(H-H_{\mathrm{D}}\right) /\left(2 \sigma_{H}{ }^{2}\right)},
$$

where $\delta(H)$ is the Dirac delta function, the native enthalpy $H_{\mathrm{N}}=0$, the step function $\theta(H)=1$ for $H \geq 0$, and $\theta(H)=0$ for $H<0$. $\mathrm{g}_{\mathrm{D}}(\gg 1)$ and $H_{\mathrm{D}}$ are respectively the total number and average enthalpy of the denatured conformations, whereas the standard deviation $\sigma_{H}$ specifies the width of the enthalpy distribution among them (Figure 1); see Ref. 23 for details. The corresponding partition function $Q=Q_{\mathrm{N}}+Q_{\mathrm{D}}$, whose native part $Q_{\mathrm{N}}=1$ is the statistical weight of the native state, and the denatured part

$$
Q_{\mathrm{D}}(T)=\frac{\mathrm{g}_{\mathrm{D}}}{\sqrt{2 \pi} \sigma_{H}} \int d H \mathrm{e}^{-\left(H-H_{\mathrm{D}}\right) /\left(2 \sigma_{H}^{2}\right)} \mathrm{e}^{-H /\left(k_{B} T\right)}
$$

Hence $[\mathrm{D}]=Q_{\mathrm{D}} / Q$. We perform numerical integrations over $H$ to obtain thermodynamic averages such as native and denatured populations [Eq. (8)], average enthalpy, and heat capacity as functions of temperature, from which the midpoint temperatures and $\kappa$ 's defined above are determined. To simplify these calculations, rather than integrating through $H \rightarrow+\infty$, we use a high $H$ cutoff that set $\mathrm{g}(H)=0$ for $H>4 H_{\mathrm{D}}$ in Eq. (7). The special case of a strictly two-state model (corresponding to $\left.\sigma_{H} \rightarrow 0\right)$ is discussed in the Appendix.

For the class of models we study, we fix both the average enthalpy $\left(H_{\mathrm{D}}\right)$ and entropy (parametrized by $\left.\mathrm{g}_{\mathrm{D}}\right)$ of the denatured state. This leads ${ }^{23}$ to an essentially constant $\Delta H_{\text {cal }}=H_{\mathrm{D}}$. Only the denatured enthalpy distribution width $\sigma_{H}$ is varied. Here we use $H_{\mathrm{D}} / k_{B}=3 \times 10^{4}$ (equivalent to $H_{\mathrm{D}}=60.0 \mathrm{kcal} \mathrm{mol}^{-1}$ ), and $\mathrm{g}_{\mathrm{D}}=5.68 \times 10^{38}$ (Figure 1$)$. These values are the same as those used in our previous study. ${ }^{23}$ They correspond approximately to the experimental data obtained by Jackson et al. ${ }^{54}$ for the Ile $\rightarrow$ Val76 mutant of chymotrypsin inhibitor 2 (CI2; see Fig. 3 of Ref. 54). Hence we believe that realistic protein energetics can be explored using this class of models.

Figure 2 shows how the model midpoint temperatures and thermodynamic cooperativity vary with $\sigma_{H}$. The calorimetric two-state criterion allows for some tolerance. This is because even small single-domain proteins deviate slightly from a strictly two-state description, ${ }^{33}$ with $\Delta H_{\mathrm{vH}} / \Delta H_{\text {cal }}$ slightly less than unity. So we do not have to require model $\Delta H_{\mathrm{vH}} / \Delta H_{\text {cal }}$ to be exactly equal to unity. Nonetheless, it is also clear that the experimental observation of $\Delta H_{\mathrm{vH}} / \Delta H_{\text {cal }} \approx 1$ imposes severe constraints on enthalpy distributions in proteins. Experimentally, $\Delta H_{\mathrm{vH}} / \Delta H_{\text {cal }}=0.96$ is reported by Fersht and coworkers $^{54}$ for CI2, other calorimetric two-state proteins have similar $\Delta H_{\mathrm{vH}} / \Delta H_{\text {cal }}$ 's (Ref. 33.) For the present models, if the $\Delta H_{\mathrm{vH}} / \Delta H_{\text {cal }}$ 's are to be $\geq 0.96$, it requires $\sigma_{H} \leq 775$ (Figure $2 \mathrm{~b}$, in units of $k_{B}$ ). This means a very narrow denatured enthalpy distribution, as the standard deviation $\sigma_{H}$ has to be less than or equal to $775 /\left(3 \times 10^{4}\right) \approx 1 / 40$ of the average enthalpic separation between the native and the denatured states, $\Delta H_{\text {cal }}$ (see Figure 1). Within this class of models, thermodynamic stability correlates with cooperativity (Figure 2a). For $\Delta H_{\mathrm{vH}} / \Delta H_{\mathrm{cal}} \approx 1$, the folding transition temperature $\approx 65^{\circ} \mathrm{C}$ corresponds to that observed experimentally. ${ }^{54}$ However, stability decreases as the denatured enthalpy 
distribution widens. The transition temperature falls below $0^{\circ} \mathrm{C}$ when $\sigma_{H}$ exceeds $\approx 1 / 17$ of $\Delta H_{\text {cal }}$.

Figure 2a shows the relation among the three midpoint temperatures. They are essentially identical when the model protein is highly cooperative (small $\left.\sigma_{H}\right)$. The difference between $T_{d}$ and the other two temperatures increases as cooperativity diminishes. This is because when the enthalpy distribution in the denatured state is wide (large $\sigma_{H}$ ), there are more low-lying nonnative enthalpies, which tend to lower the overall average enthalpy. As a result, more than half of the chain population has to be denatured (hence a higher temperature than $T_{1 / 2}$ is required) to achieve an average enthalpy of $\Delta H_{\text {cal }} / 2$ than when the denatured enthalpy distribution is narrower (smaller $\sigma_{H}$ ). This accounts for the differences among the three $\kappa$ 's [Eq. (6)] and $(\kappa)^{2}$ 's in Figures $2 \mathrm{c}$ and d. For real two-state proteins, $T_{d}$ can differ from $T_{\max }$ by $\sim 1^{\circ} \mathrm{C}$ (Ref. 50 ). On the other hand, $T_{\max }$ is practically identical to $T_{1 / 2}$ for a much wider range of cooperativity for these models. It appears that $T_{\max } \approx T_{1 / 2}$ is a consequence of $\mathrm{g}_{\mathrm{D}} \gg 1$. Model proteins with less conformational freedom ${ }^{23}$ than those considered in Figures 1 and 2 have non-negligible differences between $T_{1 / 2}$ and $T_{\max }$ (see Appendix and discussions on three-dimensional lattice models below).

Figures 2c and d compare the population-based ${ }^{23} \kappa_{0}=$ $\Delta H_{\mathrm{vH}} / \Delta H_{\text {cal }}$ with experimental formulas and their variations. For this class of models, $\kappa_{0}=\kappa_{1}=\kappa_{2}$ holds almost exactly. Owing to the behavior of $T_{d}$ discussed above, $\kappa_{3}$ deviates from the other three $\kappa$ 's when the model is not cooperative, but all four $\kappa$ 's are practically identical if their values are $\geq 0.9$. When the enthalpy ratios $\kappa$ 's are less than one, naturally the square-root $(\kappa)$ formulas Eq. (6) gives larger van't Hoff to calorimetric enthalpy ratios than the $(\kappa)^{2}$ formulas. The latter equate $\Delta H_{\mathrm{vH}}$ with $4 k_{B} T_{\text {midpoint }}^{2} C_{P}\left(T_{\text {midpoint }}\right) / \Delta H_{\text {cal }}$ (Ref. 22,40,41,51). However, when any one of the $\Delta H_{\mathrm{vH}} / \Delta H_{\text {cal }}$ 's equals unity, it implies that all other $\Delta H_{\mathrm{vH}} / \Delta H_{\text {cal }}$ 's also equal unity.

These observations suggest that the following general conclusion should be valid: Insofar as a protein can be fully denatured by heat ${ }^{23}$ (as these models are), which implies that it has a sufficiently high denatured-state entropy relative to the native state (which should be satisfied by all proteins because of their polymeric nature), all of the $\Delta H_{\mathrm{vH}} / \Delta H_{\text {cal }}$ 's considered in this paper provide essentially the same calorimetric two-state conditions, and

\footnotetext{
${ }^{2}$ Solvent-mediated (effective) intraprotein interactions can have enthalpic as well as entropic contributions. However, heat-induced conformational changes would be impossible if these interactions do not contain enthalpic parts. The interaction energy $E$ was taken to be purely enthalpic in Onuchic et al.'s random-energy treatment of temperature dependences
}

thus have the same requirement on the density of states of the proteins.

Recently, Zhou et al. ${ }^{22}$ used a homopolymer tetramer model to show that it is possible to have $\left(\kappa_{3}\right)^{2}>1$, and that the deviation from the calorimetric criterion is not simply related to the population with intermediate enthalpies. Remarkably, the thermodynamic properties of their continuum tetramer model are very similar ${ }^{23}$ to that of a lattice tetramer toy model introduced previously by Dill et al. ${ }^{4}$ Since the ground-state populations of these small systems are substantial ${ }^{23}$ even under athermal conditions $(T=\infty)$, they cannot be fully "denatured." Hence this interesting and important observation of Zhou et al. is not inconsistent with our general conclusion regarding proteins. The present study does not address the application of van't Hoff analysis to chemical reactions in solutions ${ }^{55}$ because of fundamental differences between chemical reactions and the conformational transition of polymeric systems treated here.

\section{Calorimetric two-state cooperativity implies a very low "glass transition" temperature for the folding of two-state proteins.}

The above thermodynamic results are relevant to folding kinetics, especially landscape theories that utilize the spin-glass approach put forth in the seminal work of Bryngelson and Wolynes. ${ }^{56,57}$ It has been argued, and has been generally accepted, that in order for a protein to fold in a kinetically efficient manner, its folding transition temperature $T_{\mathrm{f}}$ must be significantly greater than a glass temperature $T_{\mathrm{g}}$ that characterizes the onset of sluggish folding kinetics as the temperature is lowered ${ }^{58}$ (reviewed in Refs. 3, 4). Subsequently, based on a series of insightful studies by Onuchic, Wolynes and coworkers, ${ }^{45,59,60}$ it has been further argued that a "law of corresponding states" $6,59,60$ can be used to predict the ratio $T_{\mathrm{f}} / T_{\mathrm{g}}$ for real proteins from simulations of a 27 mer 3-letter code (3LC) model protein configured on three-dimensional cubic lattices ${ }^{45,59}$ (see discussion below). This approach provided an estimate of $T_{\mathrm{f}} / T_{\mathrm{g}}=1.6$ for small $\alpha$-helical proteins. ${ }^{6,42,43,59}$ More recently, Onuchic et al. ${ }^{9}$ considered the thermodynamics of a Gaussian random energy model similar to the one employed here and derived the relation $T_{\mathrm{f}} / T_{\mathrm{g}}=\left(H_{\mathrm{D}} / \sigma_{H}\right) \sqrt{2 / \operatorname{lng}_{\mathrm{D}}}$ (in the present notation). ${ }^{2}$ 
The estimate $T_{\mathrm{f}} / T_{\mathrm{g}} \approx 1.6$ was based on kinetic simulations. As such, it may be viewed as a lower bound for a protein to satisfy a certain requirement for foldability. A previous random-energy-model analysis already suggests that a higher thermodynamic $T_{\mathrm{f}} / T_{\mathrm{g}}$ ratio may be needed to satisfy the additional constraint imposed by calorimetric two-state cooperativity. ${ }^{23}$ Figure $2 \mathrm{~b}$ shows calorimetric cooperativity as a function of $T_{\mathrm{f}} / T_{\mathrm{g}}$ (the horizonal axis is marked by the inverse of this ratio, $T_{\mathrm{g}} / T_{\mathrm{f}}$, by applying Eq. (12) of Onuchic et al. ${ }^{9}$ ). Using realistic protein parameters, ${ }^{23,54}$ Figure $2 \mathrm{~b}$ shows that in the context of the present random-energy model analysis, for a protein's $\Delta H_{\mathrm{vH}} / \Delta H_{\text {cal }}>0.96$ (Ref. 54), it is necessary for $T_{\mathrm{f}} / T_{\mathrm{g}}>5.8 ; \Delta H_{\mathrm{vH}} / \Delta H_{\text {cal }}>0.99$ implies $T_{\mathrm{f}} / T_{\mathrm{g}}>10.0 ;$ and $T_{\mathrm{f}} / T_{\mathrm{g}} \approx 1.6$ would imply that the protein is not calorimetrically two-state, with $\Delta H_{\mathrm{vH}} / \Delta H_{\text {cal }}<0.2$.

Therefore, combining our results with Onuchic et al.'s analysis ${ }^{9}$ leads us to the conclusion that for proteins that are calorimetrically two-state, $T_{\mathrm{f}} / T_{\mathrm{g}}$ should be higher than the earlier estimate of 1.6, and may well exceed 6.0. In that case, even for an hypothetical highly stable two-state protein with $T_{\mathrm{f}} \approx 100^{\circ} \mathrm{C}(373.15 \mathrm{~K}), T_{\mathrm{g}}$ is still very low, at $\approx 62 \mathrm{~K}$. This folding glass transition temperature is a theoretical construct for quantitating a "rugged" landscape's impediment to the kinetics of folding from the denatured to the native state. The physics it describes is different from the "glass transition" of native proteins observed experimentally at $\approx 200 \mathrm{~K}$ (see, for example, Ref. 61), though it has been suggested $^{59}$ that the two phenomena might be related. The present calorimetric estimate of $T_{\mathrm{g}} \approx 62 \mathrm{~K}$ is much lower than temperatures at which folding actually takes place. While the idealized enthalpy distribution of a random-energy model without explicit chain representation might have underestimated the chance of having low-enthalpy kinetic traps, such traps should nevertheless be improbable given this extremely low estimate for $T_{\mathrm{g}}$. Therefore, our results suggest that in general kinetic traps should have at most minimal effects on the folding of real calorimetrically two-state proteins of sizes comparable to CI2. ${ }^{19,37,42,43}$ This view is apparently supported by recent folding experiments on proteins with no kinetic intermediates. ${ }^{62-67}$ In this perspective, it would be particularly revealing to elucidate the relationship between multi-phasic kinetics and calorimetric cooperativity for real proteins that do fold with kinetic intermediates (see, for example, Refs. 68-70, and theoretical perspectives in Refs. 3-8, and 11).

Lattice protein models: Why compare them against the calorimetric two-state criterion?
We now turn to protein models with explicit chain representations. Recent years have seen sustained efforts in using highly simplified lattice models to understand general properties of proteins. Lattice protein models were pioneered by Gō and coworkers. ${ }^{15} \mathrm{Go}$ models assume that only those contact interactions that occur in the native conformation can be favorable, whereas all nonnative interactions are neutral. This approach to modeling may be characterized as teleological, because the native conformation is hardwired explicitly into the model potential function. A lot of useful insight has been gained by this methodology. But it is important to realize that a Go model leaves open the question as to what physical interactions can conspire to produce the remarkable molecular recognition effect it has assumed.

An essential difference between Gō models and models introduced in the past decade - beginning with the simplest 2-letter HP potential, ${ }^{30,32}$ is that many of the more recent models have adopted microscopic interaction schemes that are independent of a particular native conformation. Therefore, these models offer the possibility to better explore the physico-chemical bases of protein folding. While much have been learnt (see, for example, Refs. 1-9, 11, 12), the goal of using these models to elucidate general protein properties has not been fully realized. One of the most generic thermodynamic properties of many small single-domain proteins is their calorimetric two-state cooperativity. However, no three-dimensional lattice model has been evaluated against the calorimetric two-state criterion. We do so here for six representative models. This was motivated by a previous study of two-dimensional models, ${ }^{23}$ which has led us to suspect that to design a physically plausible three-dimensional interaction scheme to reproduce calorimetric two-state behaviors might be non-trivial, and that other deficiencies of lattice models in describing real two-state protein properties $^{37}$ might be intimately related to their lack of calorimetric two-state cooperativity.

We take this as the first step in an endeavor to build simple tractable self-contained models to capture more proteinlike features. It is hoped that once models are required to better conform to the calorimetric two-state criterion, mechanisms for other two-state proteinlike properties would either be apparent or become more easily decipherable. From this vantage point, the substantial amount of lattice model data accumulated over the years constitutes a valuable repository of information. By applying appropriate experimental tests on these models for their similarities with and their differences from real protein behaviors, one would gain new insight into what

that leads to Eq. (12) in Ref. 9. 
novel energetic ingredients might be necessary for building better models.

We consider six models, ${ }^{44-49}$ as shown in Figure 3. We choose to analyze these models in depth because they are representative and instructive, covering a varieties of approaches and assumptions employed in recent efforts to model proteins as chains configured on three-dimensional simple cubic lattices. Some models in Figure 3 have been studied extensively and contributed significantly to the advances in theoretical understanding. All these models are based upon additive pairwise nearest-latticeneighbor contact energies. As described in the original references, ${ }^{44-49}$ the contact energies are all assumed to be temperature independent. We therefore refer to these energies as enthalpies, as in Ref. 23, to conform to the terminology in the experimental calorimetric literature.

\section{Lattice simulation methods.}

Using the model potential functions described in their respective original studies, ${ }^{44-49}$ thermodynamic quantities of these models were computed using standard Metropolis Monte Carlo (MC) histogram techniques. ${ }^{71,72}$ The chain move set we used consists of end, corner, and crankshaft moves, as described by Socci and Onuchic, ${ }^{44}$ with additional sidechain moves for the 20letter sidechain model (Figure 3f).$^{48}$ Each histogram was computed using a total of $4.5 \times 10^{8}$ attempted moves, whereby data was collected after allowing for an initial equilibrating run of $5 \times 10^{7}$ attempted moves. Every attempted move is counted as elapsed MC time in computing Boltzmann averages, whether it is accepted or rejected; and if rejected, regardless of whether it is caused by excluded volume violation or by the stochastic Metropolis algorithm for an attempted move that involves a finite increase in energy (enthalpy). The simulation temperatures are given in the captions for Figures 4-9. In one case (the Gō model in Figure 7), we also performed several independent MC simulations at different temperatures to confirm the MC histogram results.

\footnotetext{
${ }^{3}$ For the 20-letter model, the temperature at which the Boltzmann average $\langle\mathbf{Q}\rangle$ of the number of native contact $\mathbf{Q}$ equals one half of the total number $\mathbf{Q}_{\mathrm{N}}$ of native contacts was reported to be 0.272 in Ref. 49 (note that this $\mathbf{Q}$ is different from the symbol $Q$ for partition function), whereas the present simulation gives 0.279 . The discrepancy is not big. However, it is not clear whether the discrepancy merely reflects numerical uncertainties or is it related to a possible systematic deviation from the correct Boltzmann distribution in previous simulations in which attempted moves rejected by excluded volume violations were not counted as elapsed MC time (page 185 of Ref. 49, page 1617 of Ref. 73), as has been noted recently (Ref. 47).
}

Our sampling of the densities of states should be adequate since we obtained essentially the same midpoint temperatures as the original studies for all six models. ${ }^{3}$

Thermodynamic functions relevant to calorimetric considerations are plotted in Figures 4-9. In these figures, $T_{1 / 2}$ is the temperature at which the chain population $[\mathrm{N}]$ in the single lowest-enthalpy conformation equals $1 / 2$. This single-lattice-conformation definition of the model native state and the corresponding identification of $T_{1 / 2}$ with the folding transition temperature coincide with the original formulations in four of the models. ${ }^{44-47}$ However, a multiple-lattice-conformation native state containing other conformations in addition to the lowest-enthalpy conformation was advocated by the authors of the two 20-letter models. ${ }^{48,49}$ Hence, according to their definitions, the "native" populations in their models ${ }^{48,49}$ are larger than $[\mathrm{N}]$ in Figures 6 and 9. We will give more detailed consideration to the issue of native state definition below.

\section{Evaluating lattice protein models against the calorimetric two-state criterion.}

\section{A First Step: Modeling Heat Capacity Functions With No Baseline Subtractions}

We first apply the model heat capacity and enthalpy functions in Figures 4-9 directly to the relation ${ }^{23} \kappa_{0}=$ $\left\langle\Delta H\left(T_{1 / 2}\right)\right\rangle_{\mathrm{D}} / \Delta H_{\text {cal }}$ and Eq. (6) above to compute various $\Delta H_{\mathrm{vH}} / \Delta H_{\text {cal }}$ ratios in Table II. This is equivalent to assuming that for each model (as for the randomenergy models above), the entire model $C_{P}$ function is directly comparable to the "transition" part of an experimental excess heat capacity function, ${ }^{41}$ the analyses of which has led to the calorimetric two-state condition $\Delta H_{\mathrm{vH}} / \Delta H_{\mathrm{cal}} \approx 1$ for many small proteins. Experimentally, the transition part of the excess heat capacity is obtained by performing baseline subtractions on the raw data. ${ }^{23,41}$ This exercise we now undertake is a necessary and instructive starting point that involves minimal 
assumption, ${ }^{23}$ as it does not entail performing any baseline subtraction on model results. After a basic perspective has been gained, we will discuss in a later section the feasibility and appropriateness of applying baseline subtractions to model specific heat functions.

In addition to the $C_{P}$ functions, the upper panels of Figures 4-9 also show the heat capacity contributions $\left(C_{P}\right)_{\mathrm{D}}[\mathrm{D}]$ from thermal transitions among nonnative (in these cases, non-ground-state) conformations. ${ }^{23}$ When a large fraction of $C_{P}$ arises from transitions among nonnative conformations instead of transitions between native (N) and nonnative (D) conformations, significant deviations from calorimetic two-state behaviors by the $\kappa_{0} \approx 1$ standard are expected ${ }^{23}$ (Table II). This is because a large $\left(C_{P}\right)_{\mathrm{D}}[\mathrm{D}]$ contribution means that even after passing the denaturation transition midpoint (when $[\mathrm{D}]>1 / 2$ ), the average denatured enthalpy will continue to rise substantially when the temperature is further raised (see the lower panel of Figure 4, for example), as denatured chains are propelled to populate conformations at higher and higher enthalpies. Table II summarizes the six models' conformity to calorimetric two-state criteria based on different $\Delta H_{\mathrm{vH}}$ 's. Calorimetric cooperativities measured by common experimental $\Delta H_{\mathrm{vH}} / \Delta H_{\text {cal }}$ formulas such as $\left(\kappa_{2}\right)^{2}$ and $\left(\kappa_{3}\right)^{2}$ (see Table I) can readily be calculated from Table II.

None of the Models Tested Meets the Calorimetric TwoState Standard

Table II shows that all six models tested by the present method do not meet the experimental calorimetric twostate standard. Among them, the Go model appears to be most cooperative, with $\kappa_{0}=0.54$ and $\kappa_{2} \approx \kappa_{3}=0.87$. If the common experimental formulas $\left(\kappa_{2}\right)^{2}$ (Ref. 41) and $\left(\kappa_{3}\right)^{2}$ (Ref. 51) for van't Hoff to calorimetric enthalpy ratio are used, this translates into $\Delta H_{\mathrm{vH}} / \Delta H_{\text {cal }}$ $\approx 0.75$ for this particular Go model. This is still low when compared with experimental values of $\approx 0.96$ (Ref. 54) for calorimetrically two-state proteins. For five small compact globular proteins - ribonuclease A, lysozyme, $\alpha$-chymotrypsin, cytochrome $c$, and metmyoglobin, Privalov ${ }^{51}$ reported an average $\Delta H_{\mathrm{vH}} / \Delta H_{\text {cal }}=$ $\left(\kappa_{3}\right)^{2}=0.96 \pm 0.03$.

Different Calorimetric Criteria are Related to Definitions of the Native State — 20-Letter Models

For the models tested, the $\Delta H_{\mathrm{vH}} / \Delta H_{\text {cal }}$ values $(\kappa$ 's) vary considerably depending on what definition of van't Hoff enthalpy is used (Table II). The variation is mildest for the 2- and 3-letter models, for which the populationbased $\kappa_{0}$ is almost identical to one of the experimental square-root formulas, $\kappa_{3}$. And while $\kappa_{2}$ 's are different from $\kappa_{3}$ 's for these two models, they are only $27-38 \%$ larger than $\kappa_{0}$. For the other four models, the difference between $\kappa_{0}$ and the experimental formulas $\kappa_{2}$ or $\kappa_{3}$ is larger: $\kappa_{3}$ is $1.6-1.8$ times $\kappa_{0}$ for the Go and modified HP models, whereas $\kappa_{3}$ is $\approx 7$ times bigger than $\kappa_{0}$ for the two 20-letter models. For the latter four models, however, $\kappa_{2}$ is virtually identical to $\kappa_{3}$.

The differences among $\kappa$ 's are often related to differences in the midpoint temperatures used to define them. For the 2- and 3-letter models (Figures 4 and 5), the temperature $T_{1 / 2}$ for the population-based $\kappa_{0}$ (and $\kappa_{1}$ ) are well within the peak region of the specific heat capacity function and quite close to the temperature $T_{\max }$ for $\kappa_{2}$. This accounts for the relative small differences among $\kappa_{0}$, $\kappa_{1}$, and $\kappa_{2}$ in these models. The difference between $\kappa_{0}$ and $\kappa_{2}$ is larger for the Gō and modified HP models, but $T_{1 / 2}$ still lies within the peak region of the $C_{P}$ function and not that far away from $T_{\max }$ (Figures 7 and 8). The difference between $\kappa_{0}$ and $\kappa_{2}$ is much larger for the two 20-letter models. In these constructs, $T_{1 / 2}$ is well outside the peak region of $C_{P}\left(T_{1 / 2} \ll T_{\max }\right.$, see Figures 6 and 9 ). On the other hand, $T_{\max } \approx T_{d}$ for the Go model and the 20-letter model without sidechains (Figures 6 and 7), hence they have $\kappa_{2} \approx \kappa_{3}$.

The large temperature differences between $T_{1 / 2}$ and $T_{\max }$ in Figures 6 and 9 highlight one peculiar feature of the two 20-letter models which is qualitatively different from the other four models. For both of them, the population $[\mathrm{N}]$ of the single ground-state conformation is below $10 \%$ at $T_{\max }$, whereas the $C_{P}$ at $T_{1 / 2}$ (when $[\mathrm{N}]=$ $1 / 2$ ) is very low. This feature is intimately related to the rationale for adopting a multiple-lattice-conformation native state in these models. ${ }^{48,49}$ In physical terms, it means that $C_{P}$ is dominated at low temperatures by transitions among the single ground-state conformation and other conformations with very low (close to groundstate) enthaplies, most of these conformations belong to these models' multiple-conformation native state as defined by their authors ${ }^{48,49}$ (see below). When the temperature is raised, population in the single ground-state conformation continues to decrease as more of it is being transferred to other low-enthalpy conformations. Therefore, when the temperature reaches $T_{\max }$, contributions to the peak value of $C_{P}$ are dominated by transitions between the group of low-enthalpy conformations as a whole with the large number of high-enthalpy conformations. By that time the population $[\mathrm{N}]$ in the single ground-state conformation has become quite insignificant. This is the basic reason why $\kappa_{2} \gg \kappa_{0} \approx \kappa_{1}$ for these two models (Table II).

Model Heat Capacity Functions can be Compared Directly with Experiments - Gō and 20-Letter Mainchain Models are More Cooperative

By considering random-energy models, we have argued above that all common calorimetric criteria using different $\kappa$ 's are essentially equivalent when $\Delta H_{\mathrm{vH}} / \Delta H_{\text {cal }} \approx 1$ 
and the native state is represented by a single enthalpy value in an effective density of states that describes the transition part of an experimental excess heat capacity function after proper baseline subtractions. ${ }^{23}$ The behavior of the two 20-letter models prompts us to ask a more general question: Which $\kappa$ computed from a model would be most relevant for comparing theory with experiment when $\Delta H_{\mathrm{vH}} / \Delta H_{\text {cal }}$ deviates significantly from unity and the native state of the chain model may have multiple enthalpy levels?

From an operational standpoint, among the $\Delta H_{\mathrm{vH}} / \Delta H_{\text {cal }}$ 's considered, $\kappa_{2}, \kappa_{3},\left(\kappa_{2}\right)^{2}$, and $\left(\kappa_{3}\right)^{2}$ are most directly related to experiments. This is because they can be determined by analyzing the model $C_{P}$ function alone (which corresponds to an experimental calorimetric scan) without involving an a priori definition of the "native state" (whereas such a definition is needed to determine $T_{1 / 2}$ for $\kappa_{0}$ and $\kappa_{1}$ ). It is also prudent to not commit prematurely to a general singlelattice-conformation definition of the native state.

By this operational standard, the 20-letter model without sidechains is second most cooperative after the Gō model, with $\kappa_{2} \approx \kappa_{3}=0.66$. On the other hand, the 2-letter, 3-letter and modified HP models are far from being calorimetrically two-state by all standards considered here: none of their $\kappa$ 's exceeds 0.46 ; in fact they are often much lower (Table II). In these models, at any one of the transition midpoints, the average enthalpic difference $\langle\Delta H(T)\rangle_{\mathrm{D}}$ between the denatured state and the single native conformation is low relative to $\Delta H_{\text {cal }}$ (lower panels of Figures 4, 5, 9).

2- and 3-Letter Models are Less Cooperative - "Variable Two-State" Does Not Equal "Calorimetrically TwoState"

For the 2-letter model in Figures 3a and 4, a previous study has shown that its denatured enthalpy distribution is a broad shifting peak whose center position is moving continuously to higher values as temperature is increased (for example, the peak $H \approx-64$ at $T=1.26$ whereas the peak $H \approx-16$ at $T=5.00$, see Fig. 5 of Ref. $72, H$ is equivalent to their $E$ ). Therefore, this 2-letter example corresponds to the "variable two-state" case of Dill and Shortle (Fig. 1B of Ref. 74) with heat (increasing temperature) as the "denaturing agent." The observation here implies that the variable two-state scenario can differ substantially from a calorimetric two-state transition if it entails significant post-denaturational shifting of the enthalpy distribution among the denatured conformations. The present calorimetric analysis agrees with previous assessments $^{75}$ that the 3-letter model is more cooperative (has larger $\kappa$ 's, Table II) than the 2-letter model, though both are far from being calorimetrically twostate. We will consider the 3-letter model in more detail below. The modified HP model in Figures 3 e and 8 was motivated by considerations of hydration effects. Its potential function is based on two residue types ( $\mathrm{H}$ and $\mathrm{P})$, with novel features ${ }^{47}$ such that it effectively interpolates between the standard HP potential ${ }^{30,32}$ (when chain conformations are open) and the "AB" potential ${ }^{76-78}$ (when chain conformations are compact). In the $\mathrm{AB}$ potential, like residues attract and unlike residues repel. Repulsive interactions ${ }^{19,77}$ of the $\mathrm{AB}$ type facilitate sequence design and enhance kinetic foldability in this modified model relative to the standard HP model, ${ }^{47}$ though it is insufficient for calorimetric two-state cooperativity (Table II). It is interesting to note that the spatial organization of residues in the native conformation of this modified HP model (Figure 3e) is dictated mainly by the $\mathrm{AB}$ potential. Consequently, the two types of residues are segegrated to opposite sides of the structure to minimize contact, rather than organizing into a hydrophobic $(\mathrm{H})$ core surrounded by polar $(\mathrm{P})$ residues as in typical HP ground-state conformations. ${ }^{79}$

\section{Short 20-letter Sidechain Models are not Calorimetically Cooperative}

We have also calculated Klimov and Thirumalai's ${ }^{48}$ cooperativity parameter $\Omega_{c}$ by extending the MC histogram technique to compute the temperature dependence of their structural overlap function $\chi$ (Refs. 23, 48). The results are included in Table II. While $\Omega_{c}$ is basically a measure of the sharpness of a transition and does not always correlate with the degree of conformity to calorimetric two-state cooperativity, ${ }^{23}$ for these six models the rank ordering of the three most cooperative models by $\kappa_{2}$ coincides with their rank ordering by $\Omega_{c}$. This suggests that $\Omega_{c}$ may correlate reasonably well with calorimetric cooperativity if the conformational entropies of the chain models in question are similar. The calorimetric cooperativity as measured by $\kappa_{2}$ and $\kappa_{3}$ of the 15 mer 20-letter sidechain model of Klimov and Thirumalai ${ }^{48}$ is low (Figures $3 \mathrm{f}$ and 9, their "sequence A"), and is comparable to that of the 2-letter, 3-letter, and the modified HP model. Remarkably, by the $\Omega_{c}$ measure, it is by far the least cooperative among the six models. We have also completed the same analysis for their other sidechain model, "sequence B." The results are similar $\left(\kappa_{2}=0.25\right.$, other data not shown). The low levels of calorimetric cooperativity in these sidechain models may be a consequence of the shortness of the chains, as it has been observed that models with sidechains on average have higher $\Omega_{c}$ 's than non-sidechain models with the same number of mainchain monomers. ${ }^{48}$ Nonetheless, the present results mean that how sidechains may enhance thermodynamic cooperativity in longer chain models is a question that remains to be ascertained.

The Enthalpy Distribution of Gō Model is Trimodal 
We now take a closer look, as an example, at how the underlying enthalpy distribution of the Go model (Figures $3 \mathrm{~d}, 7$ ) gives rise to its relatively high cooperativity by the calorimetric criterion. Figure 10 shows that the Gō model enthalpy distribution is very different from that of models with much lower cooperativities, such as the 2-letter model of Socci and Onuchic. ${ }^{44}$ The enthalpy distribution of the 2-letter model in Figures 3a and 4 is bimodal - the lower mode peaks at the ground-state native enthalpy $(-84)$ and encompasses enthalpies $<-77$, whereas the higher mode has a shifting peak, corresponding to a temperature-dependent variable enthalpy distribution in the denatured ensemble (Fig. 5 of Ref. 72; see above). In contrast, the denatured enthalpy distribution of the Go model consists of two widely separated peaks (Figure 10), the lower one is at $H=-54$ and the higher one is around $H=-6$ to -4 . Together with the native population at $H=-57$, these give rise to a trimodal distribution of enthalpy. (The native peak is not shown in Figure 10.)

The data in Figures 7 and 10 implies that the heat denaturation of the Go model takes place in the following manner. At low temperatures, $T<0.5$ for example, $>95 \%$ of the chain population is in the single native conformation (Figure 3d). As temperature is raised to $T=0.65-0.70$, a fraction of the native population is transferred to a group of low-enthalpy conformations with $H$ 's around -54 (Figure 10). There is an enthalpy (energy) gap of 3 units between the ground state and the lowest-enthalpy $(H=-54)$ nonnative conformations. Using MC histogram techniques, we estimated that there are $\sim 10^{5}$ nonnative conformations with $H<-44$. (For this Go model, the number of native contacts $\mathbf{Q}=-H$.) The heat capacity associated with these initial thermal transitions is small in comparison with the heat absorption peak because of the relatively narrow enthalpy differences between the native and the low-enthalpy nonnative conformations. As temperature continues to increase to $\approx T_{1 / 2}=0.75$, chains start to unfold substantially, and a concentration of population at very high enthalpies $(H \approx-6)$ begins to develop. This temperature coincides with the sharp peak of the heat capacity function (Figure 7, upper panel), which reflects the large-enthalpy thermal transitions from both the single ground-state conformation $(H=-57)$ and the low-enthalpy nonnative conformations $(H \approx-54-$ $-40)$ to the large number of high enthalpy conformations around $H \approx-6$. There are non-vanishing chain populations at enthalpy levels intermediate between the two nonnative peaks, but they are not appreciable at any temperature. When the temperature is raised further to $T=0.83-0.95$, the population at the single ground-state and the low-enthalpy nonnative conformations greatly diminishes and practically all the chains have enthalpies above $H=-16$.
Why is the Go Model More Cooperative Than Others?

Several features of this process contribute to the Gō model's relatively high cooperativity. First, unlike the 2-letter model discussed above, the population peak of high-enthalpy conformations is quite insensitive to temperature: it shifts by merely $\approx 2$ enthalpy units, from $H \approx-6$ to -4 , when the temperature is increased from $T=0.75$ to 0.95 (Figure 10). Second, unlike the 20-letter models whose single ground-state conformational populations become $<0.1$ when the temperature is raised to $T_{\max }$ (Figures 6, 9, see above), the population of the single-conformation Gō-model ground state remains substantial $(\approx 0.3)$ at the peak of the heat capacity function. In fact, all three transition midpoint temperatures are well within the peak region of $C_{P}$ for the Go model. And among the models tested, it is the one with both $T_{1 / 2}$ and $T_{d}$ closest to $T_{\max }$ - within $1.4 \%$ and $0.4 \%$, respectively (Figure 7, upper panel).

These observations rationalize certain differences in cooperativity between models. For instance, the Gō model is more cooperative than the 2-letter model in Figure 4 by all $\kappa$ measures in Table II. This is because the Go model's bimodal distribution of nonnative enthalpies (i.e., the denatured part of an overall trimodal distribution) implies that a larger variance in $H$ is possible, hence a higher peak value for $C_{P}$ [Eq. (2)], and therefore a larger $\kappa_{2}$, than the 2-letter model with a single shifting broad distribution of denatured enthalpies. The bimodal denatured enthalpy distribution of the Gō model also means that the average denatured enthalpy near $T_{1 / 2}$ should be approximately one half of the entire range of possible enthalpy variations. Hence $\kappa_{0}$ should be $\approx 0.5$ (Table II indeed gives $\kappa_{0}=0.54$.) This is higher than the $\kappa_{0}$ of the 2-letter model because the latter's denatured state is dominated by low-enthalpy conformations at its $T_{1 / 2}$. The Go model is more cooperative than the 20 -letter model in Figure 6. For the $\kappa_{2}$ measure, it is because at $T_{\max }$ the Go model has $\approx 3$ times as much chain population $[\mathrm{N}]$ in its single ground-state conformation as the 20-letter model. The highly specific, teleological interactions of the Gō model also lead to much smaller probabilities for intermediate enthalpies. These factors translate into the possibility of having a larger variance in enthalpy distribution, thus a higher peak $C_{P}$ value, and hence a higher $\kappa_{2}$ for the Go model than for the 20-letter model.

\section{Summary of Analysis With No Baseline Subtractions}

The analysis above has shown that none of the models tested is calorimetrically two-state, though there are wide variations in their deviation from being so. For models with relatively high cooperativities such as the $36 \mathrm{mer}$ 20-letter model and the 48mer Gō model, this conclusion is still somewhat tentative because baseline subtraction 
schemes $^{22,23}$ are yet to be explored (see below). These schemes can lead to effectively higher $\kappa$ 's (Ref. 22). However, for models that deviate far from $\Delta H_{\mathrm{vH}} / \Delta H_{\text {cal }} \approx 1$ for all van't Hoff enthalpies considered, in particular the modified HP and short sidechain models, the analysis carried out so far is already quite sufficient in establishing that they are not good thermodynamic models for real calorimetrically two-state proteins.

It is noteworthy that the present three-dimensional 48 mer Gō model is significantly more cooperative by the $\kappa_{2}$ criterion $(=0.87)$ than a two-dimensional $18 \mathrm{mer}$ Gō model studied previously $\left(\kappa_{2}=0.64\right) .{ }^{23}$ Apparently, the longer chain length, the ability to form a threedimensional core, and even the particular fold topology of the present Gō model might have contributed to its higher calorimetric cooperativity. These factors need to be better elucidated. As we have emphasized, the interactions in Gō models are highly artifical as they are not based explicitly on a set of plausible microscopic physical interactions. But Gō model results are nonetheless instructive as they may highlight intrinsic limitations to what can be achieved by contact interactions. At least in the context of an underlying flexible polymer model, the above observations on all six models suggest that there always exists conformations with enthalpies (energies) close to the ground state, even when conformational distribution is governed by the highly specific Go potential. This raises the question as to whether it is natural to group them together with the ground-state conformation $^{46}$ to define a multiple-lattice-conformation native state as advocated by the authors of 20-letter models. ${ }^{48,49}$ As will be seen below, this is a substantive physical question, not merely an issue of semantics. In fact, it is directly relevant to gaining a better physical understanding of baseline subtraction and devising more appropriate means to compare model predictions with calorimetric experiments.

Effects of discarding a part of model specific heat capacity to mimic experimental baseline subtractions.

\section{Physical Meaning of Baseline Subtractions}

As a first approximation, we have so far assumed, as in a previous study, ${ }^{23}$ that the heat capacity functions predicted by simple protein lattice models are directly comparable to the standard "transition part" of experimental excess heat capacity function. The latter were obtained from calorimetric data by subtracting a sigmoidal weighted baseline after first subtracting the buffer baseline. ${ }^{23,36,41}$ This follows from the conventional experimental interpretation ${ }^{33,36,51}$ that only the peak region of $C_{P}$ involves appreciable heat capacity contributions from thermal transitions between conformations that are both structurally and enthalpically significantly different from one another. In this conventional view, by subtracting the baselines, the heat capacity contributions discarded were essentially only those from solvation effects and small-amplitude motions of the protein, i.e., contributions that are regarded as unimportant in accounting for significant conformational changes. This assumption also underlies the standard empirical approach of using temperature-independent solvent accessible surface areas for both the folded and the unfolded states of a protein in thermodynamic analyses of calorimetric data. ${ }^{33,36}$ However, this picture does not correspond exactly to the properties of polymer protein models, which invariably predict a non-negligible heat capacity contribution from conformational transitions well above the peak $C_{P}$ transition region, though the amount of this contribution varies from model to model (see below).

There are other reasons to believe that the real physical situation may be more complex than the picture implied by our first approximation and conventional empirical interpretation of calorimetric data. Bond vector motions measured by NMR spin relaxation indicate that protein backbone fluctuations contribute $8-14 \mathrm{cal} \mathrm{mol}^{-1} \mathrm{~K}^{-1}$ per residue, ${ }^{80,81}$ and thus account for $\sim 20 \%$ of the heat capacity of an unfolded protein. On the other hand, similar measurements on the folded state of two proteins suggest that backbone fluctuations on average contribute only $0.5 \mathrm{cal} \mathrm{mol}^{-1} \mathrm{~K}^{-1}$ per residue, and account for $\sim 1 \%$ of the heat capacity of the native state. While the connection between NMR-measured bond vector motions and conformational diversity remains to be better elucidated, the huge difference in heat capacity contribution from backbone motions between the folded and unfolded states strongly suggests that the possibility of enthalpic transitions between structurally dissimilar conformations in the denatured ensemble cannot be neglected, and that conventional baseline subtractions might have discarded heat capacity contributions from these transitions.

More recently, a molecular dynamics simulation study using implicit solvent interactions also suggests that in addition to differences in solvation effects, there are significant heat capacity contributions to the difference between native and denatured baselines from noncovalent intraprotein interactions. ${ }^{38}$ While the heat capacity contributions from model vibrational motions of the covalent bonds $^{82}$ are essentially the same in the native and the denatured states, these simulations suggest that noncovalent interactions change more with temperature in nonnative conformations than in the native state. ${ }^{38}$ Owing to limited sampling, large numerical uncertainties were reported in this molecular dynamics study. Nonetheless, its prediction that on average non-solvation intraprotein interactions account for $\sim 71 \%$ of the heat capacity difference between native and denatured baselines (Table 2 of Ref. 38) appears to be consistent with the NMR experiments described above: If we perform a rough estimate based on cytochrome $c$ data (Ref. 33), and take $\sim 16-23$ 
cal $\mathrm{mol}^{-1} \mathrm{~K}^{-1}$ per residue to be typical native-denatured baseline differences, the NMR results ${ }^{81}$ suggest that $\sim 50$ $-70 \%$ of this difference may originate from the difference in backbone motions in the native vs. the denatured state, which is in the same range as the average molecular dynamics result.

From a polymer perspective, it is also intuitive to expect non-vanishing heat capacity contributions from thermal transitions between conformations at different enthalpic levels with significant structural differences even at temperatures above the peak $C_{P}$ region. Given the immense diversity in conformational structures, it is physically quite inconceivable how enthalpic diversity in the denatured ensemble can be entirely eliminated such that it behaves as if all conformations occupy only a single enthalpy level, which would have meant that all intraprotein solvent-mediated interactions in the denatured ensemble were exclusively entropic.

Among the lattice protein models evaluated here, in which we have taken all interactions to be enthalpic for simplicity, even the heat capacity function of the Gō model with relatively high calorimetric cooperativity has a long high-temperature tail (Figure 7, upper panel). This indicates that for this model, non-vanishing contributions to $C_{P}$ from conformational transitions are not negligible at high temperatures. A relatively long (native) tail extending to temperatures far lower than the peak $C_{P}$ region is also present for the two 20-letter models (Figures 6, 9, upper panels). On the other hand, in conventional analyses of calorimetric data, no such long tails are ever present to be considered in the transition part of the excess heat capacity function obtained from baseline subtractions. ${ }^{36,41,50,51,54}$ Even in calorimetric analyses of non-cooperative nonprotein homopolymers, ${ }^{83}$ their existence is routinely precluded by empirical baseline subtraction techniques. This mismatch between theoretical predictions and standard transition excess heat capacities necessitates a closer examination of the correspondence between the physical pictures emerging from polymer protein models and the conventional interpretation of calorimetric experiments.

Applying Baseline Subtractions to Model Heat Capacities Can Result in Higher Predicted Calorimetric Cooperativities

We now explore the effects of using an ad hoc empirical procedure, similar to what has been carried out on experimental calorimetric data, to eliminate both the native and denatured tails in model $C_{P}$ functions plotted in the upper panels of Figures 4-9. Physically, this exercise was motivated by our recognition, based on the evidence above, that conventional calorimetric baseline analyses might have substracted out "tail" contributions that are relevant for the evaluation of polymer model predictions. Hence, as an effort to put theoretical predictions on the same footing as the (no-tail) experimental transition excess heat capacities, we now perform baseline subtractions on model data to eliminate their tail contributions. We do expect, nonetheless, that the corresponding "tail" contributions in real experimental data are only a minor part of the heat capacity contributions discarded by conventional baseline subtraction on calorimetric measurements. There are reasons to expect that conventional interpretation is at least partially correct in that a majority of the contributions subtracted by standard baseline analyses are indeed heat capacity contributions from solvation effects and small-amplitude protein motions. For instance, the molecular dynamics simulation discussed above estimated $^{38}$ that only $\sim 11 \%$ of native-state heat capacity came from non-covalent interactions.

Following standard experimental procedures, ${ }^{50,51}$ (see also Ref. 22) baselines are constructed as plausible linear extrapolations from low temperature and high temperature parts of the $C_{P}$ function to its peak region; they are referred to as native (low temperature) and denatured (high temperature) baselines. These constructions are depicted in Figure 11 and the upper panels of Figures 12 and 13 for the six lattice protein models we have been considering. More details are described in the caption for Figure 11. Baseline subtraction has two opposite effects on the predicted calorimetric cooperativity. On one hand, it decreases the value of calorimetric enthalpy, because some areas under the $C_{P}$ curve are excluded from the integration for $\Delta H_{\text {cal }}$. This tends to increase the $\Delta H_{\mathrm{vH}} / \Delta H_{\text {cal }}$ ratio. On the other hand, it decreases the effective peak value of heat capacity. This tends to decrease the $\Delta H_{\mathrm{vH}} / \Delta H_{\text {cal }}$ ratio, as $\Delta H_{\mathrm{vH}}$ is proportional to the effective peak $C_{P}$ value or its square root. Here we define an effective post-baselinesubtraction $\Delta H_{\mathrm{vH}} / \Delta H_{\text {cal }}$ ratio by substituting the new effective peak heat capacity and effective calorimetric enthalpy into the expression for $\kappa_{2}$ in Eq. (6):

$$
\kappa_{2} \rightarrow \kappa_{2}^{(\mathrm{s})} \equiv \frac{2 T_{\max } \sqrt{k_{B} C_{P, \max }^{(\mathrm{s})}}}{\Delta H_{\text {cal }}(\mathrm{s})}
$$

Table III shows that for all six models, baseline subtractions lead to increases in apparent (effective) calorimetric cooperativity. However, both the modified HP model $\left(\kappa_{2}^{(\mathrm{s})}=0.41\right)$ and the short 20-letter sidechain model $\left(\kappa_{2}^{(\mathrm{s})}=0.54\right)$ remain very far away from being calorimetrically two-state, despite some improvements. On the other hand, the effective calorimetric cooperativities of the 2- and 3-letter models increase dramatically (from $\kappa_{2}=0.36$ and 0.46 to $\kappa_{2}^{(\mathrm{s})} \approx 0.94$ ) after large areas (thick denatured tails) under their $C_{P}$ functions have been subtracted out (Figure 11a and upper panel of Figure 12). Remarkably, the Gō model's $\kappa_{2}^{(\mathrm{s})}$ of 1.00 now meets the experimental standard. The 36 mer 20 -letter model's $\kappa_{2}^{(\mathrm{s})}$ also rises above 0.94 (upper panel of Figure 13). We will 
use the 27 mer 3 -letter and the 36mer 20-letter models to discuss the physical implications of these enhancements of apparent calorimetric cooperativity by baseline subtractions.

\section{Nonlinear "Formal Two-State" Baselines and Multiple-} Conformation Native States

Recently, Zhou et al. made a pertinent observation that any density of states can be formally decomposed into two arbitrary "states," and that its thermal behavior made to satisfy the calorimetric two-state criterion if one is willing to introduce (non-standard) nonlinear baseline subtractions. ${ }^{22}$ To gain further insight into the physical meaning of baseline subtractions, we found it instructive to contrast and compare the present empirical analysis with their construction. Here is a brief summary of their formulation (in our notation). Any partition function $Q$ can be written as a sum of a pair of partition functions for two "states," denoted here by "0" and "1"; viz., $Q(T)=Q_{0}(T)+Q_{1}(T)$. Let $\left(C_{P}\right)_{0}$ and $\left(C_{P}\right)_{1}$ be the individual heat capacities of the two states, computed from $Q_{0}$ and $Q_{1}$ respectively, and $T_{m}$ be the midpoint temperature at which the population in the two states are equal, i.e., $Q_{0}\left(T_{m}\right)=Q_{1}\left(T_{m}\right)$. Zhou et al.'s baselines are defined by the individual heat capacities: $\left(C_{P}\right)_{0}(T)$ for $T<T_{m}$ and $\left(C_{P}\right)_{1}(T)$ for $T>T_{m}$. Naturally, a calorimetric enthalpy $\Delta_{0}^{1} H_{\text {cal }}$ is defined to be the area between the $C_{P}$ curve and this baseline, and a midpoint heat capacity value $\Delta_{0}^{1} C_{P}\left(T_{m}\right) \equiv$ $C_{P}\left(T_{m}\right)-\left[\left(C_{P}\right)_{0}\left(T_{m}\right)+\left(C_{P}\right)_{1}\left(T_{m}\right)\right] / 2$. A populationbased van't Hoff enthalpy $\Delta_{0}^{1} H_{\mathrm{vH}}(T)$ is then computed using Eq. (3) above with $\theta=Q_{1}(T) / Q(T)$. Zhou et al. showed that in general $\Delta_{0}^{1} H_{\mathrm{vH}}\left(T_{m}\right)=\Delta_{0}^{1} H_{\text {cal }}=$ $4 k_{B} T_{m}^{2} \Delta_{0}^{1} C_{P}\left(T_{m}\right) / \Delta_{0}^{1} H_{\text {cal }}$ [Eqs. (3), (4), (12) and (15) of Ref. 22]. This identity, which corresponds to $\kappa_{0}=$ $\left(\kappa_{1}\right)^{2}=1$ if $T_{1 / 2}$ is formally replaced by $T_{m}$ [see Eq. (6)], means that the calorimetric two-state condition is always satisfied with this particular choice of baselines.

We have computed baselines for the six models according to this recipe ${ }^{22}$ and included them as dotted curves in Figure 11 and the upper panels of Figures 12 and 13. (In the discussion below, they are referred to simply as "nonlinear baselines.") For models that assume a singleconformation native state, ${ }^{44-47} Q_{0}=Q_{\mathrm{N}}$ and $Q_{1}=Q_{\mathrm{D}}$. For the two 20-letter models, $Q_{0}$ is constructed as the partition function for the multiple-conformation native state defined by the original authors, ${ }^{48,49}$ while $Q_{1}$ is defined to account for the rest of the conformations. These nonlinear "formal two-state" baselines are conceptually enlightening (see below), however, it is our view that they should not be used directly to evaluate protein models. The first reason is logical - since by construction they always lead to perfect agreements with the calorimetric two-state condition, using them on model systems would abolish the substantive physical question of whether polymer protein models conform to the experimental calorimetric requirements. Second, and more importantly, such baselines had not been used by experimentalists to analyze calorimetric data. For all cases studied here, these nonlinear baselines invariably subtract more from the peak $C_{P}$ region than conventional linear or weighted baselines (Figures 11-13). This means that using these nonlinear baselines on model $C_{P}$ functions would most likely lead to an effective heat capacity function that does not physically match the experimental transition excess heat capacity function, ${ }^{41}$ and thus would make it extremely difficult to conduct meaningful comparisons between theory and experiment. ${ }^{23}$

Much insight can be gained, however, by comparing the nonlinear baselines with the ad hoc empirical linear baselines we used. As the nonlinear baselines of Zhou et al. ${ }^{22}$ are guaranteed to produce perfect (apparent) calorimetrically two-state behaviors, it is not unreasonable to expect that if the linear baselines are close to the nonlinear baselines, the apparent calorimetric cooperativity predicted by the linear baselines would be high, and vice versa. This appears to hold for five out of our six cases: Relatively high apparent calorimetric cooperativities resulted from linear baseline subtractions for the 2-letter, 3-letter, and 36mer 20-letter models (Table III); and as expected their linear and nonlinear baselines are quite close (Figure 11a, upper panels of Figures 12 and 13). On the other hand, the nonlinear baselines are very far away from the empirical linear baselines used for the modified HP and the 15 mer sidechain models. Not surprisingly, their apparent calorimetric cooperativities remain low even after linear baseline subtractions (Figures 11c and $\mathrm{d}$ and Table III).

The only exception is the Gō model (Figure 11b), for which the nonlinear denatured baseline amounts to a dominant contribution to the overall heat capacity, and is very far from the empirical linear denatured baseline. Yet the Go model is the most cooperative among the models we evaluated, especially after linear baseline subtractions (Table III). The reason for this behavior is because we have taken the denatured state of this model to be the ensemble that encompasses all non-ground-state conformations. And since the enthalpy distribution among these nonnative conformations is bimodal (Figure 10), the nonlinear denatured baseline, which is the denatured heat capacity $\left(C_{P}\right)_{1}=\left(C_{P}\right)_{\mathrm{D}}$, involves large thermal transitions between the two denatured peaks. This accounts for its high magnitudes. In addition, owing to the adoption of a single-conformation native state, there is no nonlinear native baseline in the present consideration of this Gō model. On the other hand, if a multiple-conformation native state were adopted to incorporate the low-enthalpy conformations that are now being classified as denatured, it would have resulted in nonlinear baselines for both the alternately defined native and denatured states. Adoption of such a multiple-conformation native state would 
lead to the elimination of contributions to $\left(C_{P}\right)_{1}$ from large thermal transitions between the two enthalpy peaks in Figure 10, and hence a nonlinear denatured baseline with much reduced magnitudes. It is expected that the nonlinear baselines would then be much closer to the empirical linear baselines used in our analysis, and would give rise to a situation much more similar to the $36 \mathrm{mer}$ 20-letter case, to be discussed below.

For the 36mer 20-letter model (Figure 13), the (low temperature) nonlinear native baseline derived from a multiple-conformation definition ${ }^{49}$ of the native state is almost identical to the empirical linear native baseline. By construction, a nonlinear native baseline accounts for the heat capacity contribution from thermal transitions among the multiple conformations of the native state. Therefore, when an empirical linear native baseline essentially overlaps a particular nonlinear native baseline, and we use the empirical linear baseline for subtraction, we are effectively (empirically) adopting the multiple-conformation native state that underlies the construction of the given nonlinear native baseline. More generally, when empirical linear baselines for both the native and denatured states overlap significantly with their nonlinear counterparts for a particular formal two-state definition, ${ }^{22,49}$ and $T_{\max } \approx T_{m}$, as in this particular 20 letter case (Figure 13, upper panel), the empirical linear baseline subtraction scheme may be viewed as an empirical (approximate) adoption of the given formal two-state definition for the native and denatured states. Hence, it follows from the "formal two-state" consideration ${ }^{22}$ that such an empirical subtraction would lead to closer conformity to the calorimetric two-state criterion as observed here.

The 3-Letter (3LC) Model Predicts Significant PostDenaturational Chain Expansion - Comparison with SAXS Experiments

We now broaden our attention to other thermodynamic properties. Obviously, adherence to the calorimetric two-state criterion is only one of many physical properties of real two-state proteins. Therefore, to ascertain whether a model with high apparent calorimetric cooperativity is adequate for generic properties of real two-state proteins, we should also subject its other properties to further experimental evaluation. In this spirit, we now consider the 3-letter model in more detail. This model uses a single-conformation native state, ${ }^{45}$ and its apparent calorimetric cooperativity is quite high after empirical baseline subtractions, $\kappa_{2}^{(\mathrm{s})}=0.952$. Its behavior is

\footnotetext{
${ }^{4}$ Our conclusion here is based on the fact that the 3-letter model $R_{g}$ continues to increase as the temperature is raised above the peak $C_{P}$ transition region, and that this behav-
}

expected to be representative of lattice protein models that are based on additive pairwise contact energies and have small numbers of monomer (residue) types in their alphabets. For instance, in many respects the properties of the 3-letter model are similar to the 2-letter model, which also attains a high apparent calorimetric cooperativity after baseline subtractions (Table III). As discussed above, the 3-letter model is instrumental in Onuchic et al.'s $T_{\mathrm{f}} / T_{\mathrm{g}}=1.6$ estimate for small $\alpha$-helical proteins. ${ }^{59}$

One thermodynamic property accessible to experimental determination is the dimension of a protein, measured by its average (i.e., mean-square) radius of gyration $R_{g}$ as a function of temperature. Using the MC histogram method, we have computed this function for the 3-letter model (Figure 12, lower panel). It shows a very gradual post-denaturational increase (for $T>T_{1 / 2}, T_{\max } \approx 1.5$ ): Average $R_{g}$ is $\approx 30 \%$ larger at higher temperatures than its value at the high-temperature edge $(T \approx 1.8)$ of the peak $C_{P}$ transition region.

It appears that this prediction is signficantly different from experimental observations. Sosnick and Trewhella ${ }^{84}$ have used small-angle X-ray scattering (SAXS) to monitor the temperature dependence of $R_{g}$ of ribonuclease $\mathrm{A}$, one of the first few proteins shown to be calorimetrically two-state. ${ }^{50}$ They observed no systematic postdenaturational increase of $R_{g}$ under both reducing (no disulfide bonds) and non-reducing conditions. Under reducing conditions (which more closely corresponds to the present lattice chains without crosslinks), the transition temperature $\approx 51^{\circ} \mathrm{C}$. Sosnick and Trewhella observed no continuous chain expansion at temperatures higher than the relatively narrow transition region at $\sim 45-54^{\circ} \mathrm{C}$. Indeed, there was even a slight decrease in $R_{g}$ when the temperature reached $74^{\circ} \mathrm{C}$. More recently, Hagihara et al. ${ }^{85}$ used solution X-ray scattering to show that the temperature dependence of $R_{g}$ during heat denaturation of ribonuclease $\mathrm{A}$ and cytochrome $c$ can be well approximated by a strictly two-state model. Plaxco et al. ${ }^{64}$ used SAXS to monitor the dependence of $R_{g}$ of protein $\mathrm{L}$ on guanidine hydropchloride concentration. They also did not observe any trend of post-denaturational expansion.

The significant post-denaturational chain expansion predicted by the 3-letter model is directly related to a substantial heat-induced shifting of its denatured enthalpy distribution, as evident from its thick hightemperature $C_{P}$ tail. This behavior is similar to that noted above for the 2-letter model. The discrepancy between this 3-letter model's $R_{g}$ prediction and experiment ${ }^{4}$ suggests that, in spite of its relative high apparent calorimetric cooperativity after empirical baseline 
subtractions, it suffers from essential deficiencies as a model for real two-state proteins because of the broad and shifting enthalpy distribution among its denatured conformations. ${ }^{5}$ This observation is consistent with the proposal above that the ratio $T_{\mathrm{f}} / T_{\mathrm{g}}=1.6$ deduced from the 3-letter model may most likely be an underestimate for real two-state proteins.

\section{Multiple-Conformation Native State and Non-Native Contacts in the 20-Letter Model}

Finally, we examine in more detail the thermodynamic of the 36mer 20-letter model (Figures 13-15). This model has an apparent calorimetric cooperativity $\left(\kappa_{2}^{(\mathrm{s})}=0.943\right)$ similar to that of the 2- and 3-letter models (Table III). Its model potential is the basis of a large body of interesting work; ${ }^{11}$ and is expected to be representative of lattice protein models that are based on additive pairwise contact energies, with a large but finite number of monomer types in its alphabet, and a substantial fraction of the contact interactions being repulsive. ${ }^{19,72,77}$ Here it also serves to exemplify models with a multiple-latticeconformation native state. ${ }^{6}$

The lower panel of Figure 13 shows how the folding/denaturation transition of this model chain is tracked by different thermodynamic order parameters, which may correspond to different experimental probes. The population $[\mathrm{N}]$ of the single ground-state conformation begins to drop rapidly well below the $C_{P}$ peak temperature $T_{\max }$, whereas $T_{\max }$ essentially coincides with the midpoint temperatures for all other probes shown. This is consistent with the observation ${ }^{19}$ that in general the midpoint temperature for $[\mathrm{N}]$ is lower than that for $\langle\mathbf{Q}\rangle$. The measure $P(\mathbf{Q}>20)$ shows the sharpest transition, as it is a binary "formal two-state" order parameter for ior is not observed in experiments. Following this logic, if the subtraction scheme in Figure 12a is used to ensure high calorimetric cooperativity, there should be no appreciable increase in model $R_{g}$ for $T>2.2$ if the prediction is to be consistent with experiment. But this is not the case (Figure 12b). We believe this reflects the main physical difference between this model and experimental observation. We note, however, that a direct mapping of temperatures between the 3-letter model results and experiment is not possible because they are systems of very different sizes. For instance, the peak $C_{P}$ transition regions for real proteins cover a range of $10-20$ degrees (Refs. 50, 84). However, if we choose an energy unit to equate the 3 -letter model $T_{\max } \approx 1.51$ with the ribonuclease A midpoint temperature of $51^{\circ} \mathrm{C}$, the model peak $C_{P}$ transition region would translate into a temperature range of $\approx 130$ degrees.

${ }^{5}$ Incorporation of empirical baseline subtractions does not change our previous conclusion that additive hydrophobic interactions are insufficient for calorimetric two-state cooperativity. ${ }^{23}$ For the two-dimensional HP, Gō and HP+ models analyzed in Ref. 23, application of empirical baseline subtractions similar to the one used here is not sufficient for bringing their apparent van't Hoff to calorimetric enthalpy ratio close to unity. However, baseline subtractions are able to take the two new models introduced in Ref. 23 with cooperative interactions much closer to apparent calorimetric two-state behaviors: After subtraction, $\kappa_{2}^{(\mathrm{s})}=0.90$ for the new cooperative model with pure enthalpic interactions, and $\kappa_{2}^{(\mathrm{s})}=0.97$ for the model with entropic $\mathrm{HH}$ interaction in Ref. 23. The present consideration of the 3-letter model also generalizes the previous observation that HP-like nonspecific pairwise additive interactions are insufficient to account for certain generic thermodynamic properties of real two-state proteins.

${ }^{6}$ Note, however, that a single-conformation native state with a single ground-state energy $E_{\mathrm{N}}$ was used by the author of Ref. 11 to define the folding transition temperature $T_{\mathrm{f}}$ for a different lattice model in Ref. 76 . 
which a chain conformation can take only one of two values: either it is native (has $\mathbf{Q}>20$ ), or not $(\mathbf{Q} \leq 20){ }^{7}$ The order parameter $\langle\mathbf{Q}\rangle / \mathbf{Q}_{\mathrm{N}}$ shows a broader transition because there are 40 possible $\mathbf{Q}$ values for this $36 \mathrm{mer}$ chain. For this model, the temperature dependence of $\langle\chi\rangle$ correlates almost perfectly with that of $\langle\mathbf{Q}\rangle$ (see inset in the upper panel of Figure 13). These observations illustrate that the sharpness of a transition ${ }^{48}$ can vary significantly depending on the probe (order parameter), whereas the calorimetric criterion is a more fundamental measure of cooperativity ${ }^{33}$ because it directly probes the underlying density of enthalpic states. ${ }^{23}$

This 20-letter model is a better mimic of real two-state proteins than the 3-letter model in certain respects. For instance, its $R_{g}$ shows no significant post-denaturational expansion and therefore enjoys better agreement with the SAXS experiments discussed above (Figure 15, lower panel). We now briefly touch on two issues that are likely to be relevant in future assessments of the 20letter model's conformity to experimental two-state behavior. (i) Structural diversity of the native state: The 20-letter model allows for significant conformational variation (Figure 14). For this particular sequence, this leads to the prediction that the native state has a higher heat capacity contribution from main-chain-like motions than the fully unfolded state, as is evident from the higher $C_{P}$ value in the native tail region than the denatured tail region (Figure 13, upper panel). ${ }^{8}$ However, this does not appear to agree with the NMR experiments discussed above. ${ }^{81}$ (ii) The prevalence of nonnative contacts: For this model, the number of nonnative contacts undergoes a sharp transition near the heat absorption peak (Figure 15, upper panel). The average number is $>3$ at $T_{\max }$, reaches a peak $\approx 6$ at a temperature slightly higher than $T_{\max }$, then settles down gradually at a relatively high average number of $\approx 4.5$ for the high-temperature unfolded state. Recent NMR experiments show that nonnative interactions can exist in the compact denatured states of some proteins, ${ }^{87,88}$ but this phenomenon is not universal. ${ }^{89}$ If prevalence of nonnative contact is not a

\footnotetext{
${ }^{7}$ Using MC histogram technique, we estimated that there are $\sim 4.4 \times 10^{9}$ different conformations in this 20-letter sequence's $\mathbf{Q}>20$ native state. This is $>10^{4}$ times more than the $\approx 10^{5}$ low-enthalpy conformations in the Gō model (see above), notwithstanding a 48mer Gō model's total number of conformations is $\approx(4.68)^{(48-36)}=1.1 \times 10^{8}$ times that of this 36 mer model. ${ }^{86}$ This shows that if a multiple-conformation native state were to be defined for the Gō model, its conformational diversity would be much smaller than the one in this 20-letter model.

${ }^{8} \mathrm{~A}$ recent Gō-like continuum three-helix bundle model also predicts a higher heat capacity for the native state than the denatured state. ${ }^{22}$
}

generic property of denatured states of real two-state proteins, it would be important to ascertain whether the high number of nonnative contacts observed in this particular sequence reflects a general feature of its underlying 20-letter contact potential.

\section{Concluding Remarks}

We have examined the implications of calorimetric two-state cooperativity and other experimentally determined thermodynamic properties on a protein's density of enthalpic states. ${ }^{23,90}$ In general, they require a narrow enthalpy distribution among the denatured conformations, as has been recently proposed. ${ }^{23}$ Energy landscape theory ${ }^{9}$ has allowed us to make a connection between calorimetric two-state cooperativity and folding kinetics. Using an analytical random-energy energy model, we showed that the folding landscape parameter $T_{\mathrm{f}} / T_{\mathrm{g}} \approx 6.0$, which is significantly higher than a previous estimate of $\approx 1.6$ for small $(\sim 60$-residue $) \alpha$ helical proteins. ${ }^{59}$ Experimental observations of singleexponential folding without kinetic trapping for a number of small single-domain proteins 50-80 residues long with no disulfide bonds ${ }^{62-67,91-93}$ is consistent with either $T_{\mathrm{f}} / T_{\mathrm{g}} \approx 1.6$ or $\approx 6.0$. This is because for proteins with $T_{\mathrm{f}}<100^{\circ} \mathrm{C}$, both ratios imply a $T_{\mathrm{g}}$ far lower than any temperature at which folding kinetic experiments have been conducted $\left(T_{\mathrm{g}}<233 \mathrm{~K}\right.$ or $\left.<62 \mathrm{~K}\right)$. In general, the present random-energy-model results also imply that folding of all calorimetric two-state proteins should not be affected by kinetic traps. However, this does not appear to agree with experiment. Notable counter-examples include the calorimetrically two-state ${ }^{33}$ lysozyme ${ }^{94,95}$ and cytochrome $c .{ }^{96}$ This underscores an intrinsic limitation of the random-energy-model method because it is not a chain-based approach and does not address sequence-specific properties.

We have evaluated six lattice protein models against the calorimetric two-state criterion. The initial stage of our analysis treated the native state as a single lat- 
tice conformation. This was based on the assumption in conventional analyses of calorimetric data, which have identified the native state as the structure deposited in the Protein Data Bank. ${ }^{33,36}$ Therefore, as in a previous investigation, ${ }^{23}$ we first evaluated $\Delta H_{\mathrm{vH}} / \Delta H_{\text {cal }}$ ratios directly from the model $C_{P}$ functions, without any baseline subtractions (i.e., the baseline was first taken to be simply the $C_{P}=0$ axis). In this evaluation, none of the models came close to meeting the calorimetric two-state standard. This is consistent with our previous conclusion, based on two-dimensional models, that when the native state is considered to be consisting of a single conformation, pairwise additive contact interactions are insufficient for calorimetric two-state cooperativity. ${ }^{23}$

However, based on both theoretical and experimental considerations, principally data from NMR bond vector motion measurements, ${ }^{81}$ we have come to believe that it would be profitable to explore using empirical linear (nonzero) baselines to subtract out "tail contributions" from model $C_{P}$ functions so as to compare them on a more equal footing with experimental transition excess heat capacity functions. We have therefore taken the second step of incorporating empirical baseline subtractions in our model evaluation. Analysis of a 20-letter lattice model indicates that subtracting a nonzero native baseline amounts to a re-definition of the native state. Physically, the empirical subtraction operation is roughly equivalent to (i) classifying more conformations as native, (ii) including their contributions in the thermodynamic properties of a multiple-conformation native state, and (iii) excluding thermal transitions among these multiple native conformations from contributing to the subtracted heat capacity function.

After baseline subtractions, a Gō model meets the calorimetric two-state standard. However, while the teleological Gō potential is extremely useful for posting "what if" questions, ${ }^{43,46}$ whether and how it can be rationalized in terms of physically plausible interactions remains to be clarified. Among models with a finite alphabet of residue types, the apparent $\Delta H_{\mathrm{vH}} / \Delta H_{\text {cal }}$ ratio for the 36mer 20-letter model is relatively high after empirical baseline subtraction, though it still falls short of meeting the high experimental standard for two-state cooperativity. (Its $\left(\kappa^{(\mathrm{s})}\right)^{2}=0.89$, the corresponding ratio for real two-state proteins $\approx 0.96$.) Other models with smaller alphabets or shorter chain lengths either have low $\Delta H_{\mathrm{vH}} / \Delta H_{\text {cal }}$ ratios or exhibit significant postdenaturational chain expansions that appear to contradict X-ray scattering experiments. ${ }^{84,85}$ This suggests that a relative high level of interaction heterogeneity - as characterized by a larger alphabet ${ }^{11,97-99}$ and the presence of repulsive interactions ${ }^{19,72,77}$ — is necessary for more proteinlike thermodynamic cooperativity.

The low-temperature tails in the $C_{P}$ functions of the 36mer 20-letter and the Go models before baseline subtractions are direct consequences of the low-enthalpy conformational diversity embodied in the multipleconformation native state of the 36mer 20-letter model, and the existence in the Go model of $\sim 10^{5}$ conformations with enthalpies very close to its ground state. This suggests that, for flexible heteropolymer models that achieve high apparent calorimetric cooperativity with only pairwise additive contact interactions, the native state effectively defined by an empirical native baseline would inevitably involve significant conformational fluctuation (as modeled here by different discrete lattice conformations). If one assumes that this model prediction captures at least partially the properties of real proteins, this would imply that the a posteriori experimental calorimetric "native state" defined operationally by empirical baseline subtractions may involve significant conformational diversity, and therefore may be qualitatively different from the a priori single-conformation native state used in conventional interpretation. ${ }^{33,36}$

One of the main goals of this study was to ascertain the degree to which proteinlike thermodynamic cooperativity can be achieved by simple models, especially the question as to whether pairwise additive contact interactions are sufficient. This is part of an effort to delineate the extent to which existing simple protein models capture generic protein properties. ${ }^{37}$ This issue is also relevant to a related question regarding the sufficiency of contact interactions for protein structure prediction. ${ }^{100}$ Our analysis of the 36mer 20-letter model is particularly instructive. Its apparent calorimetric cooperativity is relatively high after empirical baseline subtractions. However, how well does its predicted native conformational diversity match that in real proteins remains to be further investigated, especially in view of the apparent discrepancy between NMR main-chain bond vector motion measurements and the relative magnitudes of the native and unfolded heat capacities in this model.

Conventional interpretation of calorimetric data has been premised on a single-conformation, X-ray crystalstructure-like native state. The present analysis suggests a new perspective that involves a higher degree of conformational heterogeneity, namely (i) the possibility of a multiple-conformation native state, and (ii) the possibility that conventional baseline subtractions could have masked a non-negligible post-denaturational change in chain dimension driven by thermal transitions among denatured conformations at different enthalpic levels. In this alternate scenario, the relationship between calorimetric two-state cooperativity and a protein's underlying enthalpic density of states becomes more complex. Nonetheless, if one characterizes the thermodynamics of real two-state proteins by both the calorimetric twostate criterion and the experimental observation ${ }^{84,85}$ that no significant post-denaturational chain expansion took place, one central aspect of the physical picture ${ }^{23}$ remains essentially the same: For thermodynamically twostate proteins, there is no significant post-denaturational 
shifting of the enthalpy distribution among the conformations of the denatured state relative to the average enthalpy of the (multiple-conformation) native state. On the other hand, a corresponding pre-denaturational shifting (i.e., under native conditions) does not contradict the experimental observations. This is consistent with the multiple-state picture ${ }^{101,102}$ emerging from nativestate hydrogen exchange experiments, ${ }^{103,104}$ as has been discussed. ${ }^{23}$ However, it is noteworthy that the baseline analysis in the present work does raise the possibility that parts of the structural fluctuation revealed by native-state hydrogen exchange can in principle correspond to conformational diversities that have been operationally absorbed into the baseline-defined calorimetric native state.

\section{Acknowledgments}

We thank Yawen Bai, Wayne Bolen, Julie FormanKay, Ernesto Freire, Roxana Georgescu, Lewis Kay, Ed Lattman, Themis Lazaridis, Kip Murphy, Kevin Plaxco, Nick Socci, Tobin Sosnick, María-Luisa Tasayco, and Dev Thirumalai for helpful discussions. We thank Julie Forman-Kay, Lewis Kay and José Onuchic for their critical reading of the manuscript and very helpful comments. This work was supported by grant MT-15323 to H.S.C. from the Medical Research Council of Canada.

\section{Appendix}

\section{Statistical mechanics of a strictly two state model.}

Here we describe basic thermodynamics of a strictly two-state model, which may be viewed as the $\sigma_{H} \rightarrow 0$ limit of the random-energy model given by Eq. (7) above. The simplicity of this extreme case makes it useful for further elucidating the relationship among different midpoint temperatures and van't Hoff enthalpies in the analysis of calorimetric cooperativity. The strictly two-state model is given by the partition function

$$
Q(T)=1+g_{\mathrm{D}} \mathrm{e}^{-H_{\mathrm{D}} /\left(k_{B} T\right)},
$$

where $g_{\mathrm{D}}$ in Eq. (7) is re-written as $g_{\mathrm{D}}$ to highlight that we now consider a discrete rather than a continuous density of states. ${ }^{23}$ For this model, $\Delta H_{\text {cal }}=H_{\mathrm{D}}$; and the average enthalpy

$$
\langle H(T)\rangle=\frac{g_{\mathrm{D}} H_{\mathrm{D}} \mathrm{e}^{-H_{\mathrm{D}} /\left(k_{B} T\right)}}{1+g_{\mathrm{D}} \mathrm{e}^{-H_{\mathrm{D}} /\left(k_{B} T\right)}} .
$$

It follows that the specific heat capacity

$$
C_{P}=\frac{\partial\langle H(T)\rangle}{\partial T}=\frac{H_{\mathrm{D}}^{2}}{k_{B} T^{2}} \frac{g_{\mathrm{D}} \mathrm{e}^{-H_{\mathrm{D}} /\left(k_{B} T\right)}}{\left(1+g_{\mathrm{D}} \mathrm{e}^{-H_{\mathrm{D}} /\left(k_{B} T\right)}\right)^{2}} .
$$

This functional form gives a single maximum value for $C_{P}$ at a certain $T=T_{\max }$. The relation between $T_{\max }$ and the population midpoint temperature

$$
T_{1 / 2}=\frac{H_{\mathrm{D}}}{k_{B} \ln g_{\mathrm{D}}}
$$

may be determined as follows. First, we note that the slope of the specific heat function at the population midpoint

$$
\left.\frac{d C_{P}}{d T}\right|_{T=T_{1 / 2}}=-\frac{H_{\mathrm{D}}{ }^{2}}{2 k_{B}\left(T_{1 / 2}\right)^{3}}<0 .
$$

This establishes $T_{1 / 2}>T_{\max }$ for a strictly two-state model. We then seek a good estimate of $T_{\max }$ by attempting an approximate solution to the $d C_{P} /(d T)=0$ condition - which is equivalent to the equation

$$
g_{\mathrm{D}} \mathrm{e}^{-\xi}=\frac{\xi-2}{\xi+2},
$$

where $\xi=-H_{\mathrm{D}} /\left(k_{B} T_{\max }\right)$. For $\ln g_{\mathrm{D}} \gg 1$, which is a reasonable assumption for proteins, as discussed in the text,

$$
T_{\max } \approx \frac{H_{\mathrm{D}}}{k_{B}\left[\ln g_{\mathrm{D}}+4 /\left(2+\ln g_{\mathrm{D}}\right)\right]}<T_{1 / 2} .
$$

The last inequality follows from Eq. (A4) for $T_{1 / 2}$, and confirms the conclusion we have drawn from Eq. (A5). Finally, since by Eqs. (A2) and (A4) $\left\langle H\left(T_{1 / 2}\right)\right\rangle=$ $\Delta H_{\text {cal }} / 2$, we have $T_{d}=T_{1 / 2}$. Therefore, for a strictly two-state model,

$$
T_{d}=T_{1 / 2}>T_{\max } .
$$

We now turn to the various van't Hoff to calorimetric enthalpy ratios considered in the text [Eq. (6)]. Obviously, by definition $\kappa_{0}=1$ for the strictly two-state model. Moreover, by Eqs. (A3) and (A4),

$$
2 T_{1 / 2} \sqrt{k_{B} C_{P}\left(T_{1 / 2}\right)}=H_{\mathrm{D}}=\Delta H_{\text {cal }} .
$$

Hence $\kappa_{1}=\kappa_{3}=1$ as well, because $T_{1 / 2}=T_{d}$. On the other hand,

$\kappa_{2}=2 T_{\max } \sqrt{k_{B} C_{P}\left(T_{\max }\right)} / H_{\mathrm{D}}=\sqrt{1-4\left(k_{B} T_{\max } / H_{\mathrm{D}}\right)^{2}}<1$.

However, for proteinlike systems, $H_{\mathrm{D}} \gg k_{B} T$ is expected for any $T$ between $0^{\circ}$ to $100^{\circ} \mathrm{C}$, hence $T_{1 / 2}=T_{\max }$ and $\kappa_{2}=1$ are very good approximations. For instance, if we use the parameters in the text for $H_{\mathrm{D}}$ and $g_{\mathrm{D}}$, which were motivated by experimental data on CI2 (Fig. 3 of Ref. 54), we get $T_{1 / 2}=336.190 \mathrm{~K}$, whereas $T_{\max }=336.025 \mathrm{~K}$ is only $0.17^{\circ} \mathrm{C}$ lower, and $\kappa_{2}=0.9997$. Therefore, for a strict two-state model with these proteinlike parameters, practically all three midpoint temperatures are identical, and all $\kappa$ 's are equal to one.

\section{References}


[1] Chan, H.S., Dill, K.A. Polymer principles in protein structure and stability. Annu. Rev. Biophys. Biophys. Chem. 20:447-490, 1991.

[2] Karplus, M., Shakhnovich, E. Protein folding: Theoretical studies of thermodynamics and dynamics. In: "Protein Folding." Creighton, T.E. (ed.). New York: Freeman, 1992:127-195.

[3] Bryngelson, J.D., Onuchic, J.N., Socci, N.D., Wolynes, P.G. Funnels, pathways and the energy landscape of protein folding: A synthesis. Proteins: Struct. Funct. Genet. 21:167-195, 1995.

[4] Dill, K.A., Bromberg, S., Yue, K., Fiebig, K.M., Yee, D.P., Thomas, P.D., Chan, H.S. Principles of protein folding - A perspective from simple exact models. Protein Sci. 4:561-602, 1995.

[5] Karplus, M., Šali, A. Theoretical studies of protein folding and unfolding. Curr. Opin. Struct. Biol. 5:58-73, 1995.

[6] Wolynes, P.G., Onuchic, J.N., Thirumalai, D. Navigating the folding routes. Science 267:1619-1620, 1995.

[7] Thirumalai, D., Woodson S.A. Kinetics of folding of proteins and RNA. Acc. Chem. Res. 29:433-439, 1996.

[8] Dill, K.A., Chan, H.S. From Levinthal to pathways to funnels. Nature Struct. Biol. 4:10-19, 1997.

[9] Onuchic, J.N., Luthey-Schulten, Z., Wolynes, P.G. Theory of protein folding: The energy landscape perspective. Annu. Rev. Phys. Chem. 48:545-600, 1997.

[10] Pande, V.S., Grosberg, A.Yu., Tanaka, T. Statistical mechanics of simple models of protein folding and design. Biophys. J. 73:3192-3210, 1997.

[11] Shakhnovich, E.I. Theoretical studies of protein-folding thermodynamics and kinetics. Curr. Opin. Struct. Biol. 7:29-40, 1997.

[12] Pande, V.S., Grosberg, A.Yu., Tanaka, T., Rokhsar, D.S. Pathways for protein folding: Is a new view needed? Curr. Opin. Struct. Biol. 8:68-79, 1998.

[13] Dill, K.A. Polymer principles and protein folding. Protein Sci. 8:1166-1180, 1999.

[14] Thirumalai, D., Klimov, D.K. Deciphering the timescales and mechanisms of protein folding using minimal off-lattice models. Curr. Opin. Struct. Biol. 9:197207, 1999.

[15] Taketomi, H., Ueda, Y., Gō, N. Studies on protein folding, unfolding and fluctuations by computer simulation. 1. The effect of specific amino acid sequence represented by specific inter-unit interactions. Int. J. Peptide Protein Res. 7:445-459, 1975.

[16] Kolinski, A., Galazka, W., Skolnick, J. On the origin of the cooperativity of protein folding: Implications from model simulations. Proteins: Struct. Funct. Genet. 26:271-287, 1996.

[17] Li, H., Helling, R., Tang, C., Wingreen, N. Emergence of preferred structures in a simple model of protein folding. Science 273:666-669, 1996.

[18] Hao, M.-H., Scheraga, H.A. Characterization of foldable protein models: Thermodynamics, folding kinetics and force field. J. Chem. Phys. 107:8089-8102, 1997.

[19] Chan, H.S., Dill, K.A. Protein folding in the landscape perspective: Chevron plots and non-Arrhenius kinetics. Proteins: Struct. Funct. Genet. 30:2-33, 1998.

[20] Hao, M.-H., Scheraga, H.A. Molecular mechanisms for cooperative folding of proteins. J. Mol. Biol. 277:973983, 1998.

[21] Buchler, N.E.G., Goldstein, R.A. Universial correlation between energy gap and foldability for the random energy model and lattice proteins. J. Chem. Phys. 111:6599-6609, 1999.

[22] Zhou, Y., Hall, C.K., Karplus, M. The calorimetric criterion for a two-state process revisited. Protein Sci. 8:1064-1074, 1999.

[23] Chan, H.S. Modeling protein density of states: Additive hydrophobic effects are insufficient for calorimetric twostate cooperativity. Proteins: Struct. Funct. Genet., accepted for publication (2000).

[24] Rapaport, D.C. "The Art of Molecular Dynamics Simulation." New York: Cambridge University Press, 1995.

[25] Duan, Y., Kollman, P.A. Pathways to a protein folding intermediate observed in a 1-microsecond simulation in aqueous solution. Science 282:740-744, 1998.

[26] Li, A., Daggett, V. Characterization of the transition state of protein unfolding by use of molecular dynamics: Chymotrypsin inhibitor 2. Proc. Natl. Acad. Sci. USA 91:10430-10434, 1994.

[27] Lazaridis, T., Karplus, M. "New view" of protein folding reconciled with the old through multiple unfolding simulations. Science 278:1928-1931, 1997.

[28] Li, A., Daggett, V. Molecular dynamics simulation of the unfolding of barnase: Characterization of the major intermediate. J. Mol. Biol. 275:677-694, 1998.

[29] Braxenthaler, M., Unger, R., Auerbach, D., Given, J.A., Moult, J. Chaos in protein dynamics. Proteins 29:417425, 1997.

[30] Lau, K.F., Dill, K.A. A lattice statistical mechanics model of the conformational and sequence spaces of proteins. Macromolecules 22:3986-3997, 1989.

[31] Laughlin, R.B., Pines, D., Schmalian, J., Stojković, B.P., Wolynes, P. The middle way. Proc. Natl. Acad. Sci. USA. 97:32-37, 2000.

[32] Chan, H.S., Dill, K.A. "Sequence space soup" of proteins and copolymers. J. Chem. Phys. 95:3775-3787, 1991.

[33] Makhatadze, G.I., Privalov, P.L. Energetics of protein structure. Adv. Protein Chem. 47:307-425, 1995.

[34] Ooi, T., Oobatake, M., Nemethy, G., Scheraga, H.A. Accessible surface areas as a measure of the thermodynamic parameters of hydration of peptides. Proc. Natl. Acad. Sci. USA. 84:3086-3090, 1987. Erratum: 84:6015, 1987.

[35] Livingstone, JR., Spolar, R.S., Record, M.T., Jr. Contribution to the thermodynamics of protein folding from the reduction in water-accessible nonpolar surface area. Biochemistry 30:4237-4244, 1991.

[36] Robertson, A.D., Murphy, K.P. Protein structure and the energetics of protein stability. Chem. Rev. 97:12511267, 1997.

[37] Chan, H.S. Protein folding: Matching speed and locality. Nature 392:761-763, 1998.

[38] Lazaridis, T., Karplus, M. Heat capacity and compactness of denatured proteins. Biophys. Chem. 78:207-217, 1999.

[39] Chan, H.S. Modelling protein folding by Monte Carlo dynamics: Chevron plots, chevron rollover, and non- 
Arrhenius kinetics. In: "Monte Carlo Approach to Biopolymers and Protein Folding." Grassberger, P., Barkema, G., Nadler, W. (eds.). Singapore: World Scientific, 1998:29-44.

[40] Privalov, P.L., Potekhin, S.A. Scanning microcalorimetry in studying temperature-induced changes in proteins. Meth. Enzymol. 131:4-51, 1986.

[41] Freire, E. Differential scanning calorimetry. In: "Methods in Molecular Biology, Vol. 40: Protein Stability and Folding: Theory and Practice." Shirley, B.A. (ed.). New Jersey, Totowa: Humana Press Inc., 1995:191-218.

[42] Shea, J.-E., Onuchic, J.N., Brooks, C.L. III. Exploring the origins of topological frustration: Design of a minimally frustrated model of fragment B of protein A. Proc. Natl. Acad. Sci. USA 96:12512-12517, 1999.

[43] Nymeyer, H., Socci, N.D., Onuchic, J.N. Landscape approaches for determining the ensemble of folding transition states: Success and failure hinge on the degree of frustration. Proc. Natl. Acad. Sci. USA 97:634-639, 2000.

[44] Socci, N.D., Onuchic, J.N. Folding kinetics of proteinlike heteropolymers. J. Chem. Phys. 101:1519-1528, 1994.

[45] Socci, N.D., Onuchic, J.N., Wolynes, P.G. Diffusive dynamics of the reaction coordinate for protein folding funnels. J. Chem. Phys. 104:5860-5868, 1996.

[46] Pande, V.S., Rokhsar, D.S. Folding pathway of a lattice model of proteins. Proc. Natl. Acad. Sci. USA 96:12731278, 1999.

[47] Sorenson, J.M., Head-Gordon, T. The importance of hydration for the kinetics and thermodynamics of protein folding: Simplifed lattice models. Fold. \& Des. 3:523534, 1998.

[48] Klimov, D.K., Thirumalai, D. Cooperativity in protein folding: From lattice models with sidechains to real proteins. Fold. \& Des. 3:127-139, 1998.

[49] Gutin, A.M., Abkevich, V.I., Shakhnovich, E.I. A protein engineering analysis of the transition state for protein folding: Simulation in the lattice model. Fold. \& Des. 3:183-194, 1998.

[50] Privalov, P.L., Khechinashvili, N.N. A thermodynamic approach to the problem of stabilization of globular protein structure: A calorimetric study. J. Mol. Biol. 86:665-684, 1974.

[51] Privalov, P.L. Stability of proteins. Small globular proteins. Adv. Protein Chem. 33:167-241, 1979.

[52] Lumry, R., Biltonen, R., Brandts, J.F. Validity of the "two-state" hypothesis for conformational transitions of proteins. Biopolymers 4:917-944, 1966.

[53] Freire, E., Biltonen, R.L. Statistical mechanical deconvolution of thermal transitions in macromolecules. I. Theory and application to homogeneous systems. Biopolymers 17:463-479, 1978.

[54] Jackson, S.E., Moracci, M., elMasry, N., Johnson, C.M., Fersht, A.R. Effect of cavity-creating mutations in the hydrophobic core of chymotrypsin inhibitor 2 . Biochemistry 32:11259-11269, 1993.

[55] Liu, Y., Sturtevant, J.M. Significant discrepancies between van't Hoff and calorimetric enthalpies. III Biophys. Chem. 64:121-126, 1997.

[56] Bryngelson, J.D., Wolynes, P.G. Spin glasses and the statistical mechanics of protein folding. Proc. Natl. Acad. Sci. USA 84:7524-7528, 1987.

[57] Bryngelson, J.D., Wolynes, P.G. A simple statistical field theory of heteropolymer collapse with application to protein folding. Biopolymers 30:177-188, 1990.

[58] Goldstein, R.A., Luthey-Schulten, Z.A., Wolynes, P.G. Optimal protein-folding codes from spin-glass theory. Proc. Natl. Acad. Sci. USA 89:4918-4922, 1992.

[59] Onuchic, J.N., Wolynes, P.G., Luthey-Schulten, Z., Socci, N.D. Toward an outline of the topography of a realistic protein-folding funnel. Proc. Natl. Acad. Sci. USA 92:3626-3630, 1995.

[60] Luthey-Schulten, Z., Ramirez, B.E., Wolynes, P.G. Helix-coil, liquid crystal, and spin glass transitions of a collapse heteropolymer. J. Phys. Chem. 99:2177-2185, 1995.

[61] Vitkup, D., Ringe, D., Petsko, G.A., Karplus, M. Solvent mobility and the protein 'glass' transition. Nature Struct. Biol. 7:34-38, 2000.

[62] Scalley, M.L., Baker, D. Protein folding kinetics exhibit an Arrhenius temperature dependence when corrected for the temperature dependence of protein stability. Proc. Natl. Acad. Sci. USA 94:10636-10640, 1997.

[63] Plaxco, K.W., Simons, K.T., Baker, D. Contact order, transition state placement and the refolding rates of single domain proteins. J. Mol. Biol. 277:985-994, 1998.

[64] Plaxco, K.W., Millett, I.S., Segel, D.J., Doniach, S., Baker, D. Chain collapse can occur concomitantly with the rate-limiting step in protein folding. Nature Struct. Biol. 6:554-556, 1999.

[65] Chiti, F., Taddei, N., White, P.M., Bucciantini, M., Magherini, F., Stefani, M., Dobson, C.M. Mutational analysis of acylphosphatase suggests the importance of topology and contact order in protein folding. Nature Struct. Biol. 6:1005-1009, 1999.

[66] Martinez, J.C., Serrano, L. The folding transition state between SH3 domains is conformationally restricted and evolutionarily conserved. Nature Struct. Biol. 6:10101016, 1999.

[67] Riddle, D.S., Grantcharova, V.P., Santiago, J.V., Alm, E., Ruczinski, I., Baker, D. Experiment and theory highlight role of native state topology in SH3 folding. Nature Struct. Biol. 6:1016-1024, 1999.

[68] Troullier, A., Reinstädler, D., Dupont, Y., Naumann, D., Forge, V. Transient non-native secondary structures during the refolding of $\alpha$-lactalbumin detected by infrared spectroscopy. Nature Struct. Biol. 7:78-86, 2000.

[69] Schultz, C.P. Illuminating folding intermediates. Nature Struct. Biol. 7:7-10, 2000.

[70] Demarest, S.J., Raleigh, D.P. Solution structure of a peptide model of a region important for the folding of $\alpha$-lactalbumin provides evidence for the formation of nonnative structure in the denatured state. Proteins: Struct. Funct. Genet. 38:189-196, 2000.

[71] Ferrenberg, A.M., Swendsen, R.H. New Monte Carlo technique for studying phase transitions. Phys. Rev. Lett. 61:2635-2638, 1988.

[72] Socci, N.D., Onuchic, J.N. Kinetic and thermodynamic analysis of proteinlike heteropolymers: Monte Carlo histogram technique. J. Chem. Phys. 103: 4732-4744, 1995 . 
[73] Šali, A., Shakhnovich, E., Karplus, M. Kinetics of protein folding: A lattice model study of the requirements for folding to the native state. J. Mol. Biol. 235:16141636, 1994.

[74] Dill, K.A., Shortle, D. Denatured states of proteins. Annu. Rev. Biochem. 60:795-825, 1991.

[75] Socci, N.D., Nymeyer, H., Onuchic, J.N. Exploring the protein folding funnel landscape. Physica D 107:366382, 1997.

[76] Shakhnovich, E.I., Gutin, A.M. Engineering of stable and fast-folding sequences of model proteins. Proc. Natl. Acad. Sci. USA 90:7195-7199, 1993.

[77] Chan, H.S., Dill, K.A. Comparing folding codes for proteins and polymers. Proteins: Struct. Funct. Genet. 24:335-344, 1996.

[78] Bornberg-Bauer, E., Chan, H.S. Modeling evolutionary landscapes: Mutational stability, topology, and superfunnels in sequence space. Proc. Natl. Acad. Sci. USA 96:10689-10694, 1999.

[79] Yue, K., Dill, K.A. Forces of tertiary structural organization in globular proteins. Proc. Natl. Acad. Sci. USA 92:146-150, 1995.

[80] Yang, D., Kay, L.E. Contributions to conformational entropy arising from bond vector fluctuations measured from NMR-derived order parameters: Application to protein folding. J. Mol. Biol. 263:369-382, 1996.

[81] Yang, D., Mok, Y.K., Forman-Kay, J.D., Farrow, N.A., Kay, L.E. Contributions to protein entropy and heat capacity from bond vector motions measured by NMR spin relaxation. J. Mol. Biol. 272:790-804, 1997.

[82] Brooks, B., Karplus, M. Harmonic dynamics of proteins: Normal modes and fluctuations in bovine pancreatic trypsin inhibitor. Proc. Natl. Acad. Sci. USA 80:6571-6575, 1983.

[83] Tiktopulo, E.I., Bychkova, V.E., Rička, J., Ptitsyn, O.B. Cooperativity of the coil-globule transition in a homopolymer - Microcalorimetric study of poly( $N$-isopropylacrylamide). Macromolecules 27:28792882, 1994.

[84] Sosnick, T.R., Trewhella, J. Denatured states of ribonuclease A have compact dimensions and residual secondary structure. Biochemistry 31:8329-8335, 1992.

[85] Hagihara, Y., Hoshino, M., Hamada, D., Kataoka, M., Goto, Y. Chain-like conformation of heat-denatured ribonuclease A and cytochrome $c$ as evidence by solution X-ray scattering. Fold. \& Des. 3:195-201, 1998.

[86] Chan, H.S., Dill, K.A. The effects of internal constraints on the configurations of chain molecules. J. Chem. Phys. 92:3118-3135, 1990; Erratum: 107:10353, 1997.

[87] Blanco, F.J., Serrano, L., Forman-Kay, J.D. High populations of non-native structures in the denatured state are compatible with the formation of the native folded state. J. Mol. Biol. 284:1153-1164, 1998.

[88] Mok, Y.K., Kay, C.M., Kay, L.E., Forman-Kay, J. NOE data demonstrating a compact unfolded state for an SH3 domain under non-denaturing conditions. J. Mol. Biol. 289:619-638, 1999.

[89] Gillespie, J.R., Shortle, D. Characterization of longrange structure in the denatured state of staphylococcal nuclease. II. Distance restraints from paramagnetic relaxation and calculation of an ensemble of structures. J. Mol. Biol. 268:170-184, 1997.

[90] Gō, N. Theory of reversible denaturation of globular proteins. Int. J. Peptide Protein Res. 7:313-323, 1975.

[91] Jackson, S.E., Fersht, A.R. Folding of chymotrypsin inhibitor 2. 1. Evidence for a two-state transition. Biochemistry 30:10428-10435, 1991.

[92] Schindler, T., Schmid, F.X. Thermodynamic properties of an extremely rapid protein folding reaction. Biochemistry 35:16833-16842, 1996.

[93] Burton, R.E., Huang, G.S., Daugherty, M.A., Calderone, T.L., Oas, T.G. The energy landscape of a fast-folding protein mapped by Ala $\rightarrow$ Gly substitutions. Nature Struct. Biol. 4:305-310, 1997.

[94] Radford, S.E., Dobson, C.M., Evans, P.A. The folding of hen lysozyme involves partially structured intermediates and multiple pathways. Nature 358:302-307, 1992.

[95] Kiefhaber, T. Kinetic traps in lysozyme folding. Proc. Natl. Acad. Sci. USA 92:9029-9033, 1995.

[96] Sosnick, T.R., Mayne, L., Hiller, R., Englander, S.W. The barriers in protein folding. Nature Struct. Biol. 1:149-156, 1994.

[97] Wolynes, P.G. As simple as can be? Nature Struct. Biol. 4:871-874, 1997.

[98] Wang, J., Wang, W. A computational approach to simplifying the protein folding alphabet. Nature Struct. Biol. 6:1033-1038, 1999.

[99] Chan, H.S. Folding alphabets. Nature Struct. Biol. 6:994-996, 1999.

[100] Vendruscolo, M., Najmanovich, R., Domany, E. Can a pairwise contact potential stabilize native protein folds against decoys obtained by threading? Proteins: Struct. Funct. Genet. 38:134-148, 2000.

[101] Bai, Y., Englander, S.W. Future directions in folding: The multi-state nature of protein structure. Proteins: Struct. Funct. Genet. 24:145-151, 1996.

[102] Llinás, M., Gillespie, B., Dahlquist, F.W., Marqusee, S. The energetics of T4 lysozyme reveal a hierarchy of conformations. Nature Struct. Biol. 6:1072-1077, 1999.

[103] Englander, S.W., Mayne, L., Bai, Y., Sosnick, T.R. Hydrogen exchange: The modern legacy of LinderstrømLang. Protein Sci. 6:1101-1109, 1997.

[104] Li, R., Woodward, C. The hydrogen exchange core and protein folding. Protein. Sci. 8:1571-1590, 1999. 
Table I

\begin{tabular}{|c||c|c|c|c|}
\hline$T_{\text {midpoint }}$ & $\Delta H_{\mathrm{vH}} / \Delta H_{\text {cal }}$ & references & $\Delta H_{\mathrm{vH}} / \Delta H_{\text {cal }}$ & references \\
\hline \hline$T_{1 / 2}$ & $\begin{array}{c}\kappa_{0} \\
\theta=[\mathrm{D}]\end{array}$ & Ref. 23, Eq. (4) & & \\
\hline$T_{1 / 2}$ & $\kappa_{1}$ & Ref. 23 & $\left(\kappa_{1}\right)^{2}$ & Ref. 23 \\
\hline$T_{\max }$ & $\kappa_{2}$ & Ref. 40, Eq. (39) & $\left(\kappa_{2}\right)^{2}$ & $\begin{array}{l}\text { Ref. 40, Eq. (38) } \\
\text { Ref. 41, Eq. (21) }\end{array}$ \\
\hline$T_{d}$ & $\kappa_{3}$ & Ref. 50, Eq. (7) & $\begin{array}{c}\left(\kappa_{3}\right)^{2} \\
\theta=\langle\Delta H\rangle / \Delta H_{\text {cal }}\end{array}$ & $\begin{array}{l}\text { Ref. 51, Eq. (11) } \\
\text { Ref. 22, Eq. (22) }\end{array}$ \\
\hline
\end{tabular}

Table I. Different definitions in the literature for $\Delta H_{\mathrm{vH}} / \Delta H_{\text {cal }}$, the van't Hoff to calorimetric enthalpy ratio. $T_{\text {midpoint }}$ is the midpoint temperature of the given definition(s); see Eq. (6) in the text. Equation numbers in the table are those in the example reference(s) in which a given formula is used or proposed. $\theta$ 's are shown only for $\Delta H_{\mathrm{vH}} / \Delta H_{\text {cal }}$ 's that follow directly from Eq. (4). Note that $\kappa_{0}, \kappa_{2},\left(\kappa_{2}\right)^{2}$, and $\left(\kappa_{3}\right)^{2}$ are equal, respectively, to the expressions " $\Delta H_{\mathrm{vH}} / \Delta H_{\text {cal }}$," " $\Delta H_{\mathrm{vH}}^{\exp } / \Delta H_{\text {cal }}$," " $\Delta H_{\mathrm{vH}}^{\exp (\mathrm{a})} / \Delta H_{\text {cal }}$," and " $\Delta H_{\mathrm{vH}}^{\exp (\mathrm{a}) \prime} / \Delta H_{\text {cal }}$ " in Ref. 23. 
Table II

\begin{tabular}{|c|c|c|c|c|c|c|}
\hline \multirow[t]{2}{*}{ Model } & \multirow[t]{2}{*}{$\Delta H_{\text {cal }}$} & \multicolumn{4}{|c|}{$\Delta H_{\mathrm{vH}} / \Delta H_{\mathrm{cal}}$} & \multirow[t]{2}{*}{$\Omega_{c}$} \\
\hline & & $\kappa_{0}$ & $\kappa_{1}$ & $\kappa_{2}$ & $\kappa_{3}$ & \\
\hline (a) 2-letter & 68.5 & $\overline{0.26}$ & 0.32 & 0.36 & $\overline{0.24}$ & $\overline{11.2}$ \\
\hline (b) 3-letter (27mer) & 73.9 & 0.36 & 0.43 & 0.46 & 0.31 & 20 . \\
\hline (c) 20-letter (36mer) & 15.0 & 0.10 & 0.12 & 0.67 & 0.66 & 38. \\
\hline (d) Gō (48mer) & 55.2 & 0.54 & 0.78 & 0.87 & 0.87 & 192 \\
\hline (e) Modified "HP" (361 & $\overline{35.1}$ & 0.17 & 0.23 & 0.33 & 0.31 & $\overline{12.4}$ \\
\hline (f) Sidechain (15mer) & 11.6 & 0.05 & 0.07 & 0.38 & 0.36 & $\overline{5.69}$ \\
\hline
\end{tabular}

Table II. Calorimetric cooperativity of the lattice protein models in Figure 3. Thermodynamic quantities are deduced from Figures 4-9: $\kappa_{0}$ involves the population-based van't Hoff enthalpy, ${ }^{23}$ which can be readily read off from the $\langle\Delta H\rangle_{\mathrm{D}}$ curves. $\kappa_{1}, \kappa_{2}$, and $\kappa_{3}$ [Eq. (6)] are deduced from the $C_{P}$ functions, and $\Delta H_{\text {cal }}$ is obtained by numerical integration of $C_{P}$ over $T$. The Klimov-Thirumalai ${ }^{48}$ cooperativity parameter $\Omega_{c}$ is calculated for these models and included for comparison; the present $\Omega_{c}=5.69$ is slightly different from the value 5.32 reported by Klimov and Thirumalai. ${ }^{48}$ 


\section{Table III}

\begin{tabular}{|l||c|c|c|c|c|c|}
\hline Model & $T_{\max }$ & $C_{P, \max }$ & $C_{P, \max }^{(\mathrm{s})}$ & $\Delta H_{\mathrm{vH}}^{(\mathrm{s}}$ & $\Delta H_{\text {cal }}^{(\mathrm{s})}$ & $\kappa_{2}^{(\mathrm{s})}$ \\
\hline \hline (a) 2-letter (27mer) & 1.35 & 80.6 & 69.5 & 22.6 & 24.2 & 0.932 \\
\hline (b) 3-letter (27mer) & 1.56 & 117 & 105 & 32.0 & 33.6 & 0.952 \\
\hline (c) 20-letter (36mer) & 0.282 & 316 & 294 & 9.66 & 10.3 & 0.943 \\
\hline (d) Go (48mer) & 0.764 & 986 & 965 & 47.5 & 47.3 & 1.00 \\
\hline (e) Modified "HP" (36mer) & 0.558 & 107 & 102 & 11.3 & 27.8 & 0.406 \\
\hline (f) Sidechain (15mer) & 0.268 & 66.4 & 59.9 & 4.14 & 7.75 & 0.535 \\
\hline
\end{tabular}

Table III. Effects of baseline subtractions on the predicted calorimetric cooperativities of the six lattice protein models considered in this work: The effective van't Hoff to calorimetric enthalpy ratio $\kappa_{2}^{(\mathrm{s})}$ (right column) is equal to $\Delta H_{\mathrm{vH}}^{(\mathrm{s})} / \Delta H_{\mathrm{cal}}^{(\mathrm{s})}$ [Eq. (9)]. The definitions of all quantities tabulated and methods to determine them are described in the text, Figure 11, and upper panels of Figures 12 and 13. 


\section{Figure Captions}

Fig. 1 Densities of states $g(H)$ of random energy models. Each parabolic curve is $\ln g(H)$ from Eq. (7) with $H_{\mathrm{D}}=3 \times 10^{4}$ (vertical dashed lines), $\mathrm{g}_{\mathrm{D}}=5.68 \times 10^{38}$, as described in the text, and $H$ is in units of $k_{B}$. The $\kappa_{0}$ values of these curves, $0.6,0.80,0.95$, and 0.98 , quantify the different degrees of cooperativity of four models given here as examples, with standard deviations of denatured enthalpy $\sigma_{H}=1800,1350,700$, and $440 k_{B}$ respectively. $\kappa_{0}$ 's are the population-based ${ }^{23} \Delta H_{\mathrm{vH}} / \Delta H_{\text {cal }}$ ratios. The horizontal dashed line highlights the fact that for these models it is possible for $g(H)<1$; and the dot indicates that their unique native $(\mathrm{N})$ states have zero enthalpy $[\delta$-function in Eq. (7)]. Note that the logarithmic scale along the vertical axis implies that a 0.693 decrease in $\ln g$ is equivalent to halving the value of $g$ itself. Hence the distribution of $g$ is much sharper than this logarithmic plot might have otherwise conveyed.

Fig. 2 Relationship among different calorimetric twostate criteria in the random energy models defined by Eq. (7). See text and Table I for definitions and references. Left column: (a) Midpoint transition temperatures and (b) van't Hoff to calorimetric enthalpy ratios, as functions of the standard deviation $\sigma_{H}$ of denatured enthalpy distribution. (b) shows $\kappa$ 's vs. $\sigma_{H}$ times a constant, so that the horizontal scale corresponds to Onuchic et al.'s expression ${ }^{9}$ for $T_{\mathrm{g}} / T_{\mathrm{f}}$. We note that $\kappa_{0}$ in (b) is well approximated by Eq. (13) of Ref. 23. Right column: Experimental formulas for $\Delta H_{\mathrm{vH}} / \Delta H_{\text {cal }}$ vs. the population-based $\kappa_{0}$ used in our theoretical analyses.

Fig. 3 Recent three-dimensional cubic lattice protein models considered in this paper for their conformities to the calorimetric two-state criterion. Monomers (residues) are numbered from one end of the chain to the other; monomer 1 corresponds to the leftmost letter of a sequence. Each model protein chain is shown in its unique native or ground-state (lowest-enthalpy) structure. The corresponding sequence is also included, except for the Gō model in (d), as the interactions of a Gō model is determined solely by the ground-state conformation it presumes. (a) A 2-letter model of Socci and Onuchic (sequence 002 in Table 1 of Ref. 44). (b) A 3-letter model of Socci et al. (sequence in Fig. 3 of Ref. 45). (c) A 20-letter model of Gutin et al. (sequence in Fig. 1 of Ref. 49). (d) A Gō model of Pande and Rokhsar (structure in Fig. 1 of Ref. 46). (e) A modified HP "solvation" model of Sorenson and Head-Gordon (sequence 6 in Table 1 of Ref. 47). Filled and open circles represent the $\mathrm{H}$ and $\mathrm{P}$ monomers, respectively, in this modified HP model. (f) A 20-letter sidechain model of Klimov and Thirumalai (sequence A in Fig. 1 of Ref. 48). Here the main-chain monomers are numbered, and sidechains are represented by grey circles.
Fig. 4 Thermodynamic cooperativity of the 2-letter model in Fig. 3a. Results are obtained by the Monte Carlo (MC) histogram technique using simulation at $T=1.5$. $[\mathrm{N}]$ and $[\mathrm{D}]$ are respectively the fractional native and denatured population, $[\mathrm{N}]+[\mathrm{D}]=1$. In this figure and subsequent Figs. 5-9, the native state of each model is taken to be only its single ground-state (lowest $H$ ) conformation, and the denatured state consists of all other conformations. ${ }^{23}$ The vertical lines give the midpoint temperatures. From left to right, they are $T_{1 / 2}$ when $[\mathrm{N}]=[\mathrm{D}]=1 / 2($ dashed line $), T_{\max }$, and $T_{d}$ (solid lines). In all six models studied here (Figs. 4-9), $T_{1 / 2}<T_{\max }<T_{d}$. Upper panel: the specific heat capacity $C_{P}$ is defined by Eq. (2) in the text; $\left(C_{P}\right)_{\mathrm{D}}$ is the specific heat capacity of the denatured ensemble, obtained by replacing the Boltzmann averages $\langle\ldots\rangle$ in Eq. (2) over the full ensemble by averages $\langle\ldots\rangle_{\mathrm{D}}$ over the denatured (nonnative) ensemble. ${ }^{23}$ Lower panel: The excess heat function $\langle\Delta H\rangle$ (solid curve increasing with $T$ ) is given by Eq. (1) in the text, $\langle\Delta H\rangle_{\mathrm{D}}$ (dashed curve) is the corresponding average over the denatured ensemble, ${ }^{23}$ both are normalized by (in units of) $\Delta H_{\text {cal }}$ obtained by numerical integration of the entire area under the $C_{P}$ curve, part of which is shown in the upper panel. Our results for $C_{P}$ and $\langle\Delta H\rangle$ are numerically consistent with the $C_{V}$ and $\langle E\rangle$ functions in Figs. 10 and 9 of the original study. ${ }^{72}$

Fig. 5 Same as Fig. 4, but for the 3-letter model in Fig. 3b; obtained by the MC histogram technique from simulation at $T=1.5$.

Fig. 6 Same as Fig. 4, but for the 20-letter model in Fig. 3c; obtained by the MC histogram technique from simulation at $T=0.27$.

Fig. 7 Same as Fig. 4, but for the Gō model in Fig. 3d; all continuous curves are obtained by the $\mathrm{MC}$ histogram technique from simulation at $T=0.75$. For this model, $T_{\max }(0.764)$ is almost equal to $T_{d}(0.767)$. Black dots in the lower panel are fractional native populations $[\mathrm{N}]$ at six different temperatures computed by direct MC simulations, showing good agreement with results from the histogram method.

Fig. 8 Same as Fig. 4, but for the modified HP "solvation" model in Fig. 3e; obtained by the MC histogram technique from simulation at $T=0.6$. Our simulated $C_{P}$ function (upper panel) is consistent with the original simulation $\left(C_{V}\right.$ of sequence 6 in Fig. 8 of Ref. 47).

Fig. 9 Same as Fig. 4, but for the 20-letter sidechain model in Fig. 3f; obtained by the MC histogram technique from simulation at $T=0.25$. The $C_{P}$ function in 
the upper panel is consistent with the original heat capacity simulation $\left(C_{V}\right.$ in Fig. 2 c of Ref. 48$)$. Our results are also consistent with the thermodynamics properties $\langle\chi\rangle, \Delta \chi$, and $P_{\text {NBA }}$ given by Klimov and Thirumalai ${ }^{48}$ in their Fig. 2 (data not shown).

Fig. 10 Distributions of denatured (nonnative) enthalpy $H$ of the 48 mer Gō model in Figs. 3d and 7 at different temperatures $T$, obtained by direct $\mathrm{MC}$ simulations (same temperatures as the black dots in Fig. 3). The native enthalpy is -57 . The total area under a distribution curve is proportional to the fractional denatured population $[\mathrm{D}]$ at the given temperature.

Fig. 11 Exploring effects of baseline subtractions on predicted calorimetric cooperativity. Ad hoc baseline subtractions are applied to the heat capacity functions of the 2-letter (a), Gō (b), modified HP (c), and 20letter sidechain (d) models. The model heat capacities $\left(C_{P}\right.$ 's $)$ are the same as those presented in Figures 4 and 7-9. In each plot, the shaded area is subtracted from the original (pre-subtraction) $\Delta H_{\text {cal }}$ to yield a new effective calorimetric enthalpy $\Delta H_{\text {cal }}^{(\mathrm{s})}\left(<\Delta H_{\text {cal }}\right)$. Native and denatured baselines with non-zero slopes are constructed for (b) and (d). Denatured baselines with negative slopes are provided for (a) and (c), but their native baselines are assumed to have zero slope (i.e., no new native baseline) because the significant curvatures of their $C_{P}$ functions at low temperatures do not appear to warrant linear positive-slope extrapolations. Solid vertical lines mark the temperature $T_{\max }$ at the peak of heat capacity functions; the black dot marks the arithmetric mean of the values of native and denatured baselines at $T_{\max }$. Following standard experimental calorimetric baseline procedures ${ }^{50,51}$ (see also Ref. 22), the new effective heat capacity peak value $C_{P, \max }^{(\mathrm{s})}$ is given by the vertical measure between the black dot and the presubtraction $C_{P, \max }=C_{P}\left(T_{\max }\right)$. The quantities $C_{P, \max }^{(\mathrm{s})}$ and $\Delta H_{\text {cal }}^{(\mathrm{s})}$ are then used to compute the new effective van't Hoff to calorimetric enthalpy ratios $\kappa_{2}^{(\mathrm{s})}$ in Table III. Included for comparison are nonlinear "formal two-state" baselines (dotted curves) constructed using the method of Zhou et al. ${ }^{22}$ Nonlinear baselines correspond to heat capacity functions $\left(C_{P}\right)_{0}$ and $\left(C_{P}\right)_{1}$ of the native and denatured ensembles respectively. No native nonlinear baseline is provided for (a) - (c) because each of their native states is taken to have only a single conformation, as in the original analyses. ${ }^{44,46,47}$ Hence $\left(C_{P}\right)_{0}=0$ and $\left(C_{P}\right)_{1}=\left(C_{P}\right)_{\mathrm{D}}$ for $(\mathrm{a})-(\mathrm{c})$. On the other hand, for the 20-letter sidechain model in (d), the nonlinear native baseline is calculated ${ }^{22}$ from a multiple-conformation native state defined by the original authors. ${ }^{48}$ Vertical dashed lines mark the temperature $T_{m}$. For (a) $-(\mathrm{c}), T_{m}=T_{1 / 2}$; for $(\mathrm{d}), T_{m}$ is the temperature at which one half of the chain population is in the multiple-conformation native state ("native basin of attraction") defined in Ref. 48. See the text for further details.

Fig. 12 Thermodynamic/calorimetric cooperativity of a 3-letter model. (a) Same as Figure 11a, but for the 3letter model of Socci et al. ${ }^{45}$ in Figures $3 \mathrm{~b}$ and 5 . (b) Root-mean-square radius of gyration $R_{g}$ of this 3 -letter chain model vs. temperature. (Square root of the Boltzmann average of square radius of gyration of the chains.) $R_{g}$ continues to increase substantially as temperature is raised well above the transition region (vertical dashed and solid lines).

Fig. 13 Thermodynamic/calorimetric cooperativity of a 20-letter model. Upper panel: Same as Figure 11d, but for the 20-letter model of Gutin et al. ${ }^{49}$ in Figures $3 \mathrm{c}$ and 6. As in Figure 11d, the vertical dashed line marks the temperature $T_{m}$ at which one half of the chain population is in the multiple-conformation native state defined by the original authors as the ensemble of conformations that have more than 20 contacts that also occur in the ground-state conformation $(\mathbf{Q}>20, \mathbf{Q}$ is referred to as the number of native contacts). ${ }^{49}$ For this 36 mer model, the total number $\mathbf{Q}_{\mathrm{N}}$ of native contacts equals 40 . The corresponding native and denatured nonlinear baselines are calculated using the method of Zhou et al. ${ }^{22}$ Lower panel: Folding/denaturation transition tracked by different order parameters. [N] is the fractional chain population in the single-conformation ground state; $\langle\mathbf{Q}\rangle / \mathbf{Q}_{\mathrm{N}}$ is the normalized Boltzmann-averaged number of native contacts; $P(\mathbf{Q}>20)$ is the fractional population in the multiple-conformation native state; and $\langle\chi\rangle$ is the Boltzmann average of the overlap function $\chi$ of Thirumalai and coworkers, ${ }^{48}$ which is a useful measure of the structural similarity between any given conformation and the ground-state conformation. The single ground-state conformation have $\mathbf{Q} / \mathbf{Q}_{\mathrm{N}}=1$ and $\chi=0$. The inset in the upper panel shows the relation between $\mathbf{Q} / \mathbf{Q}_{\mathrm{N}}$ and $\chi$. While each $\mathbf{Q}$ is consistent with many values of $\chi$, and vice versa (scatter plot), for this model the correlation between their Boltzmann averages at different temperatures is almost perfect (curve in inset with slope $\approx-1$ ).

Fig. 14 Conformational diversity in the multiplelattice-conformation native state of the 20-letter model in Figures 3c, 6 and 13. In each conformation, the directionality of the sequence is indicated by the filled circle, which marks the position of monomer 1 in Figure 3c. The three rows show example non-ground-state conformations (from top to bottom) with number of native contacts $\mathbf{Q}=34,35$, and 36 respectively. These $\mathbf{Q}$ values are close to the average $\mathbf{Q}$ of the multiple-conformation native state at the midpoint temperatures $T_{m}$ and $T_{\max }$ 
(vertical dashed and solid lines in Figure 13). ${ }^{49}$

Fig. 15 Effects of the folding/denaturation transition on conformational properties of the 20-letter model in Figures 3c, 6 and 13. The dashed lines on the left mark $T_{1 / 2}$ at which the fractional population $[\mathrm{N}]$ of the single ground-state conformation equals $1 / 2$, the dashed lines on the right mark $T_{m} \approx T_{\max }$ (see Figure 13). Upper panel: Boltzmann-averaged number of nonnative contacts (i.e., contacts that do not belong to the single ground-state conformation) vs. temperature. Lower panel: Root-mean-square radius of gyration vs. temperature. (Same as Figure 12b, but now for the 20-letter model.) 


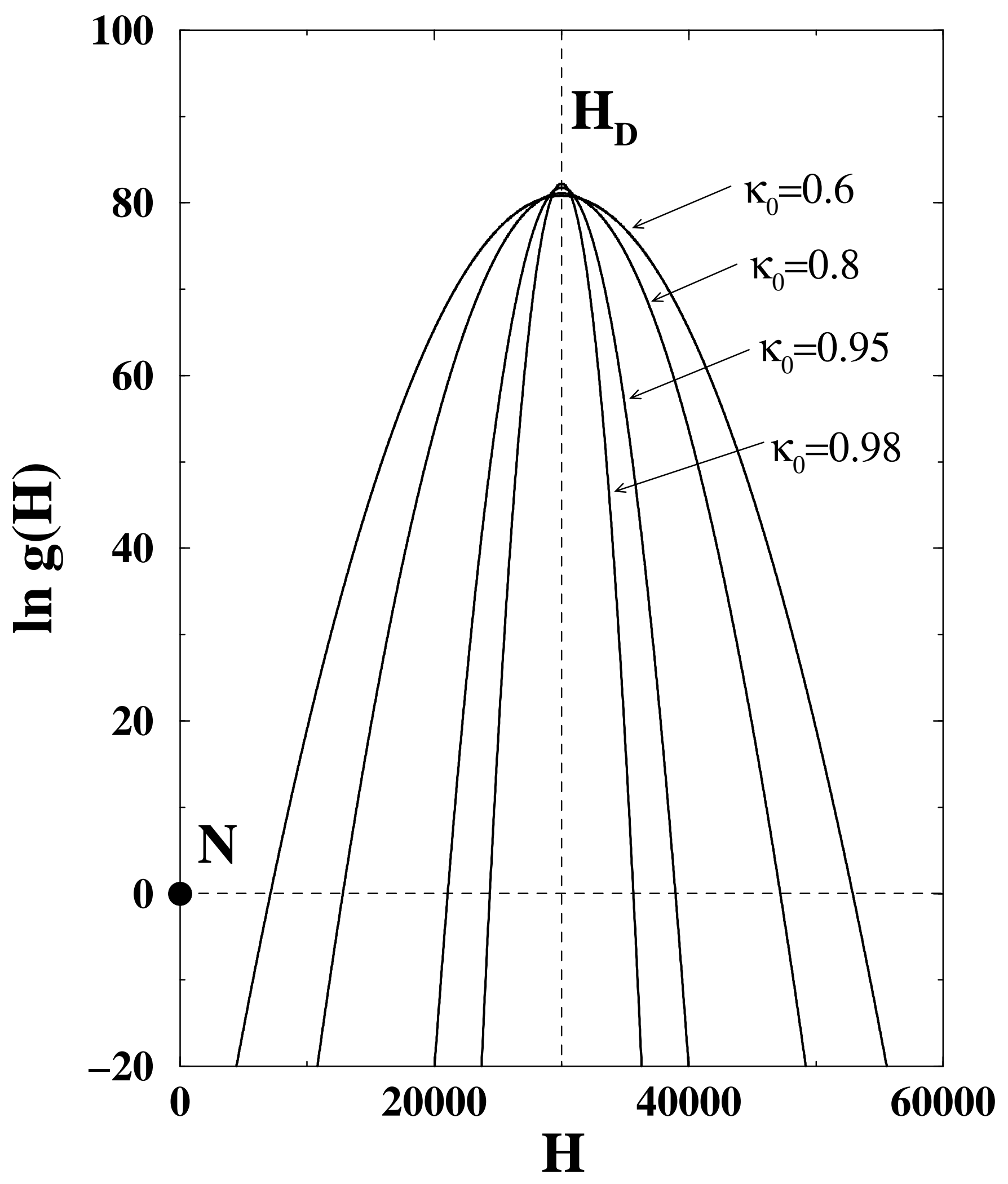



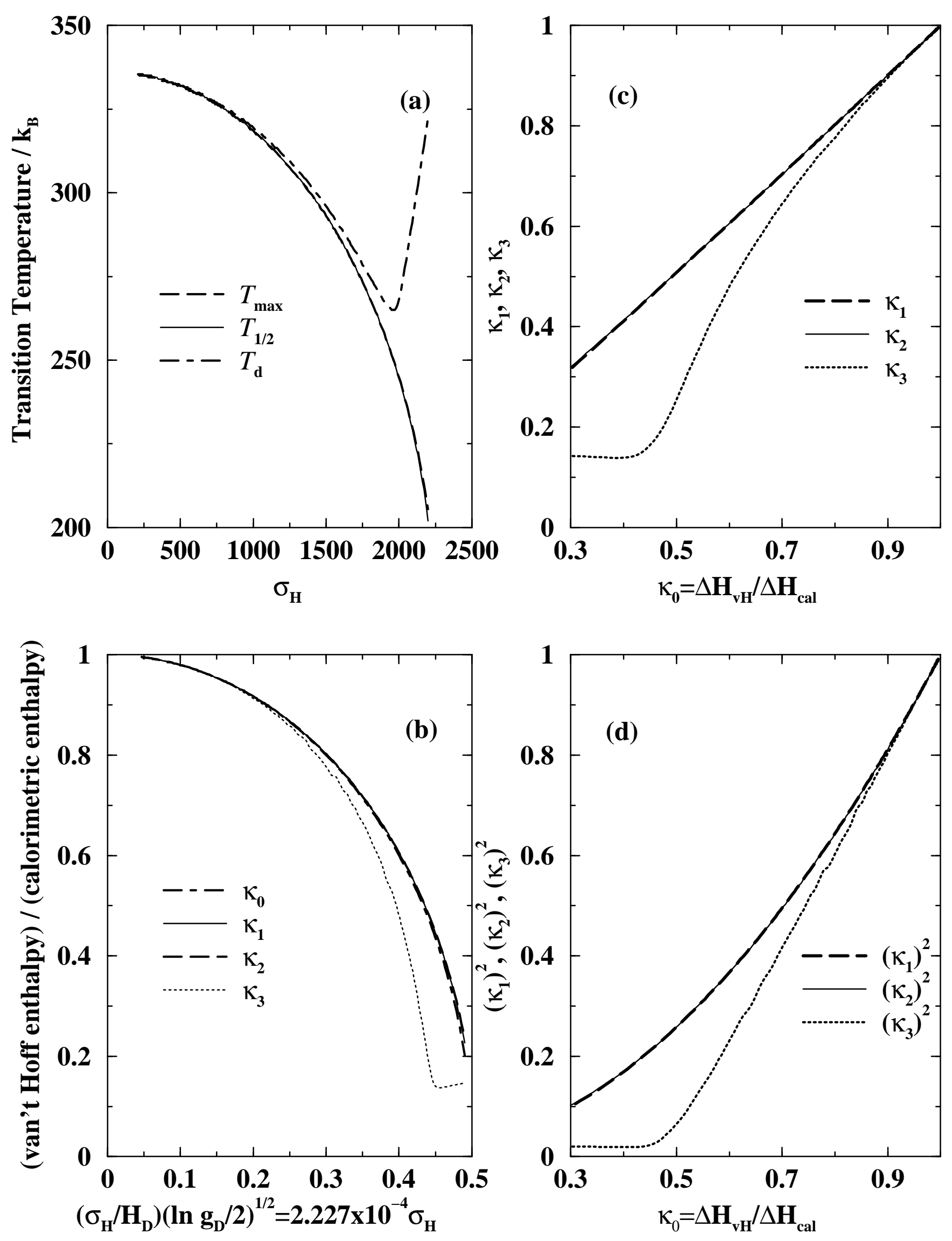


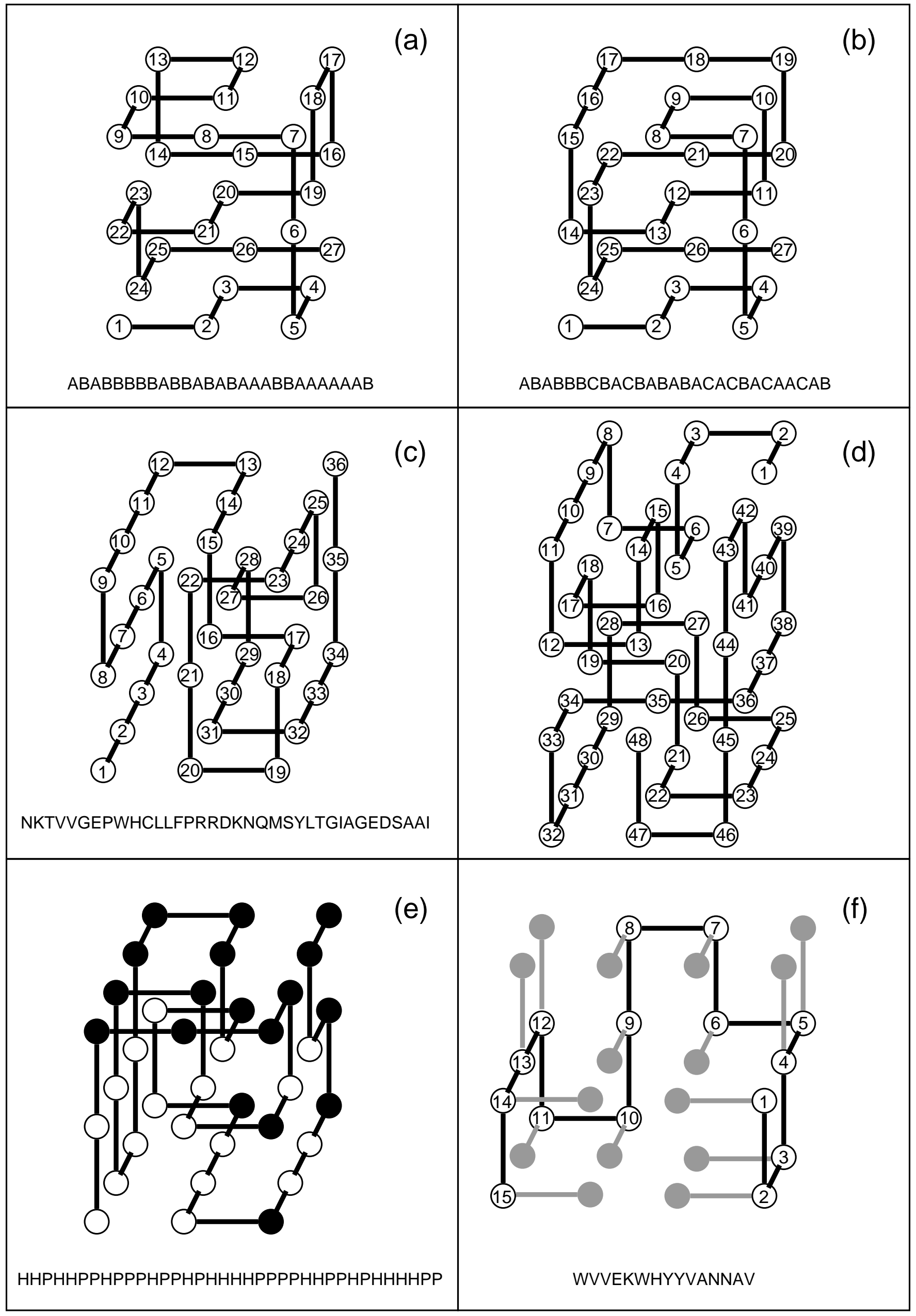



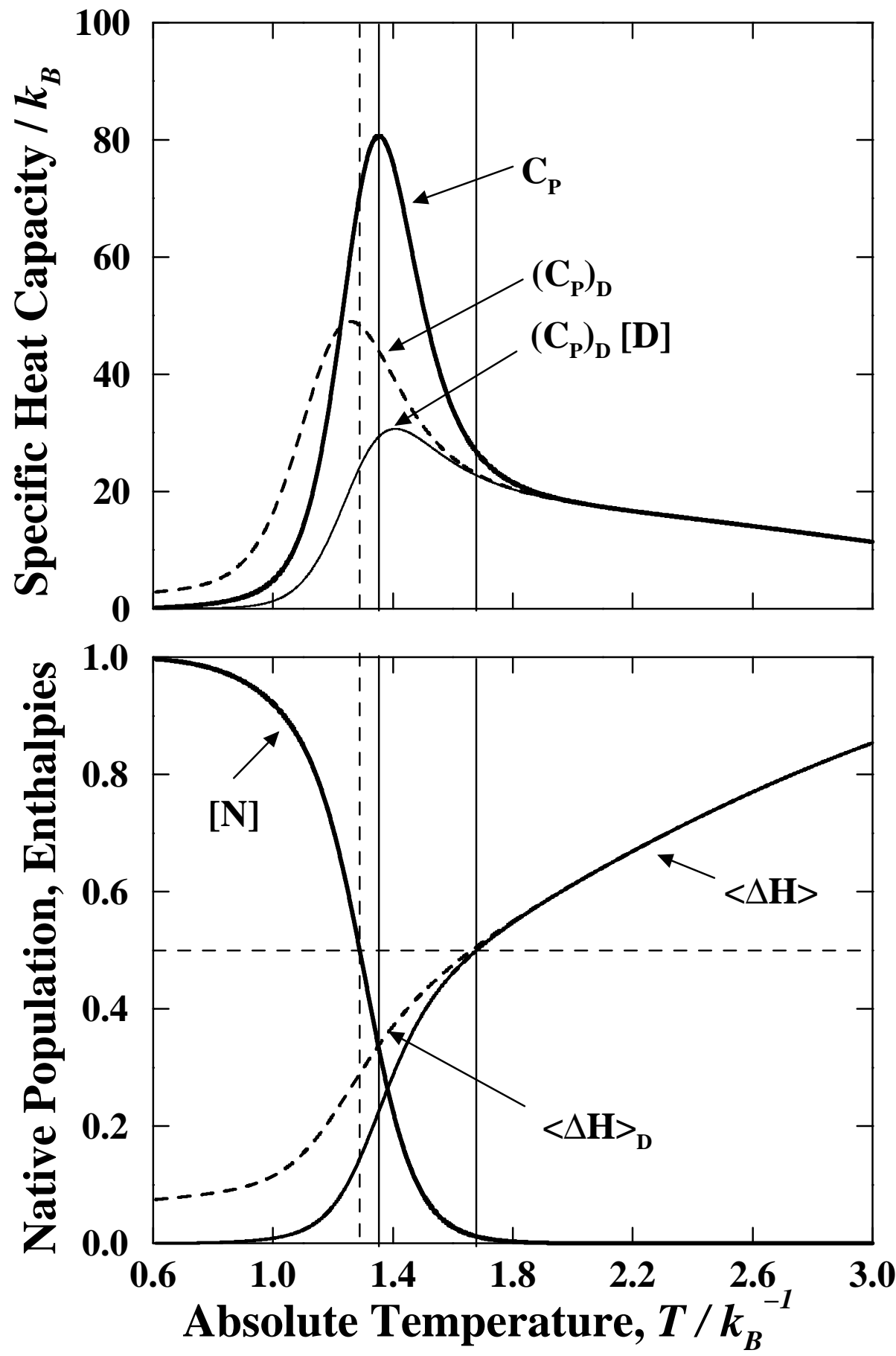

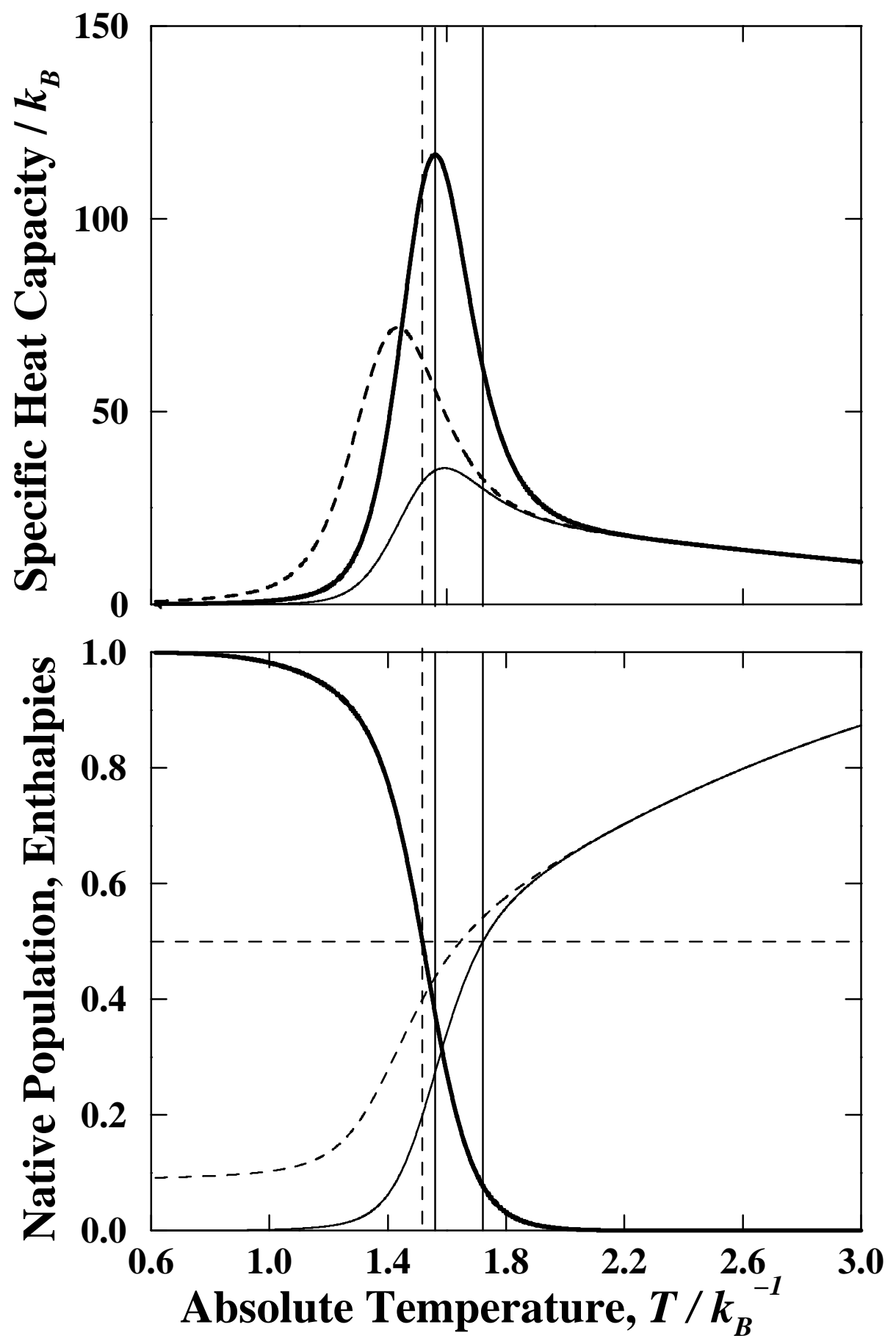

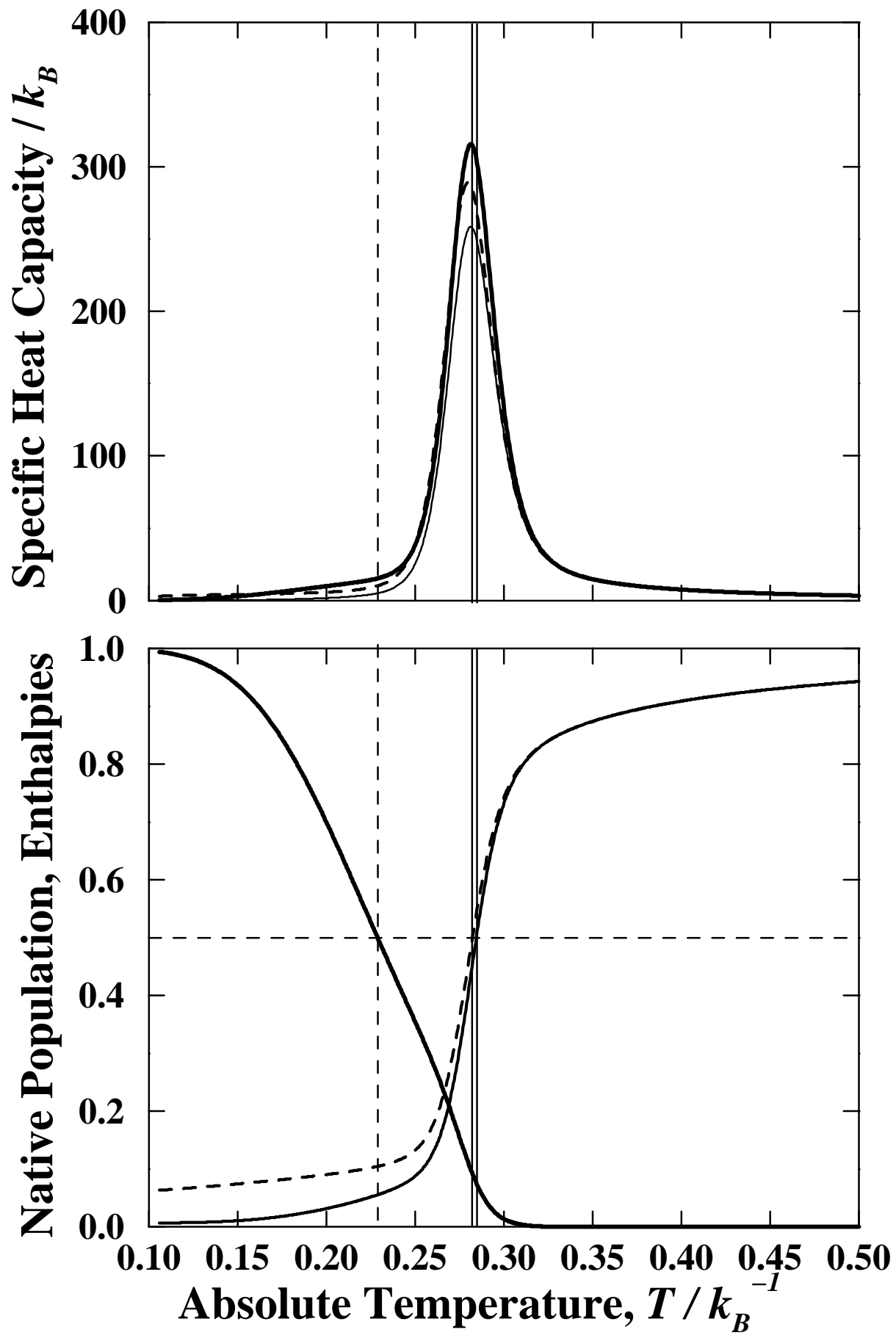

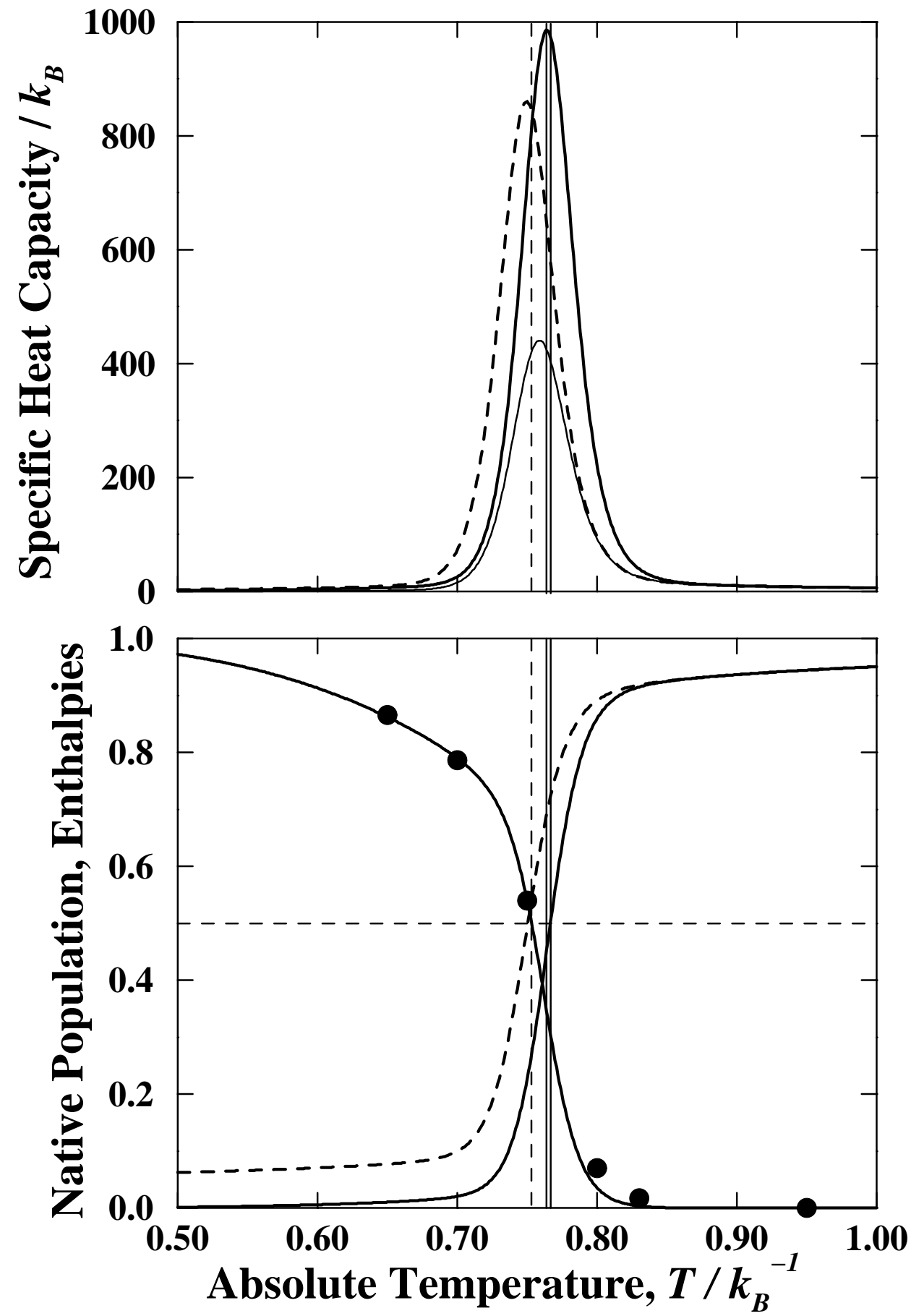

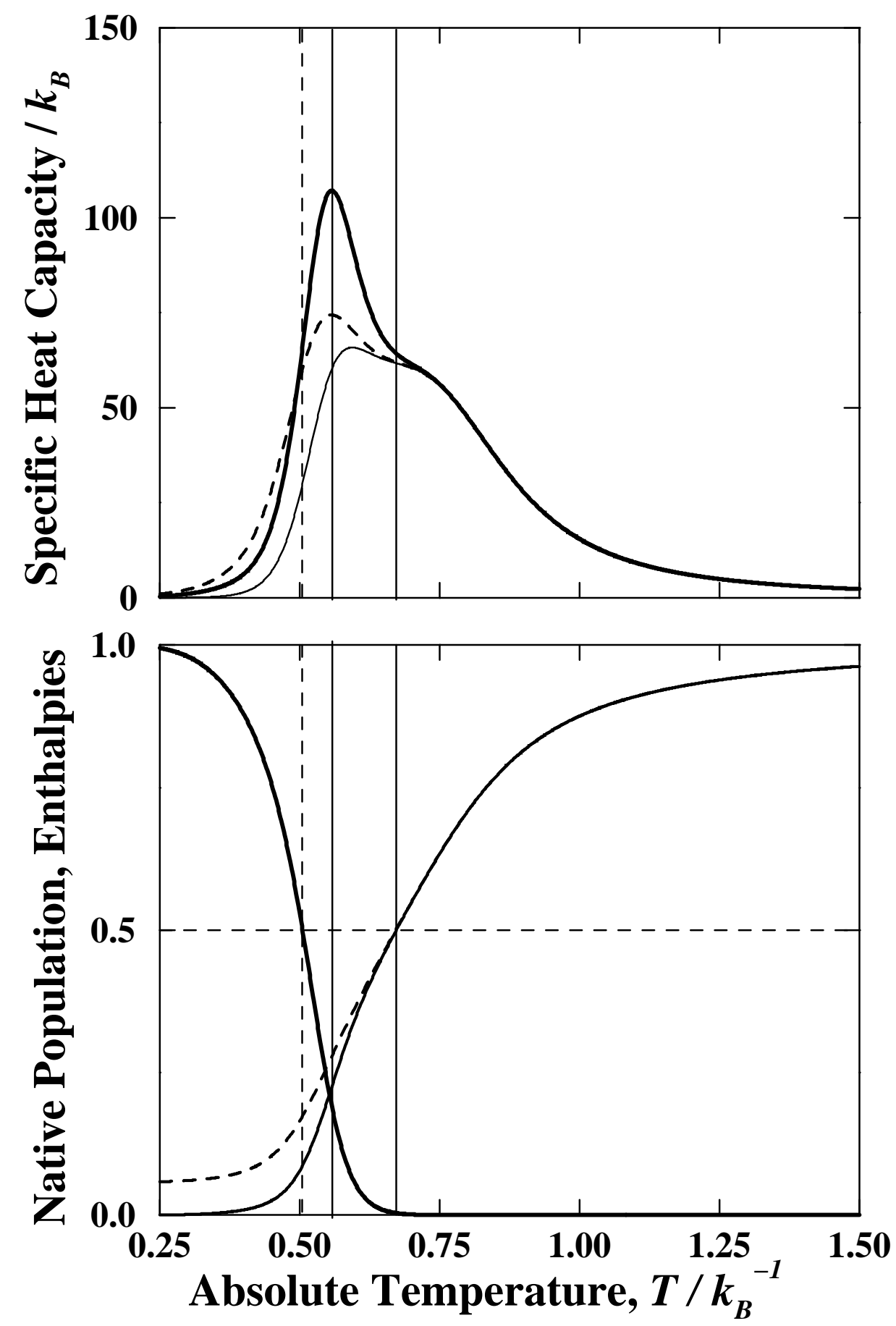

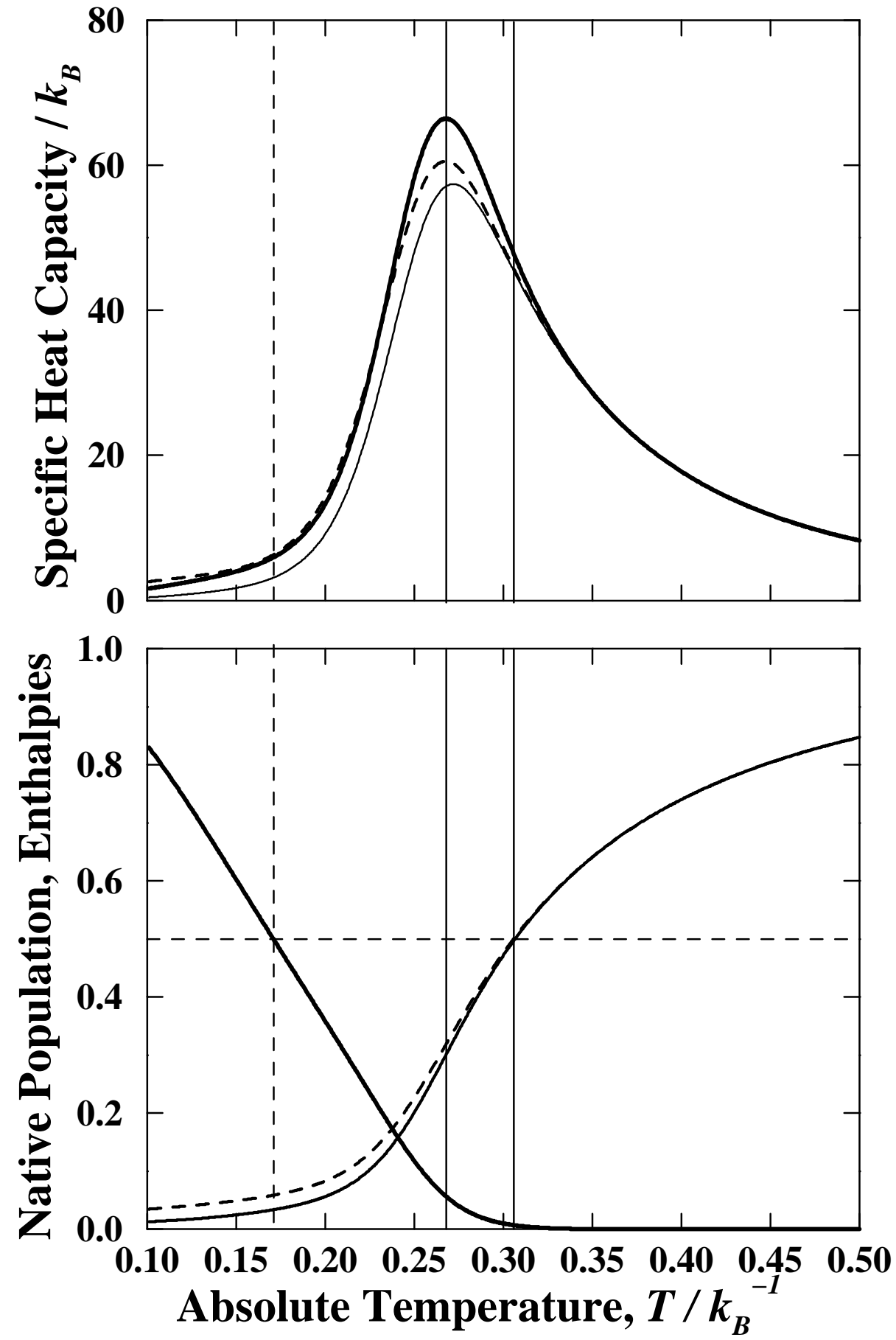


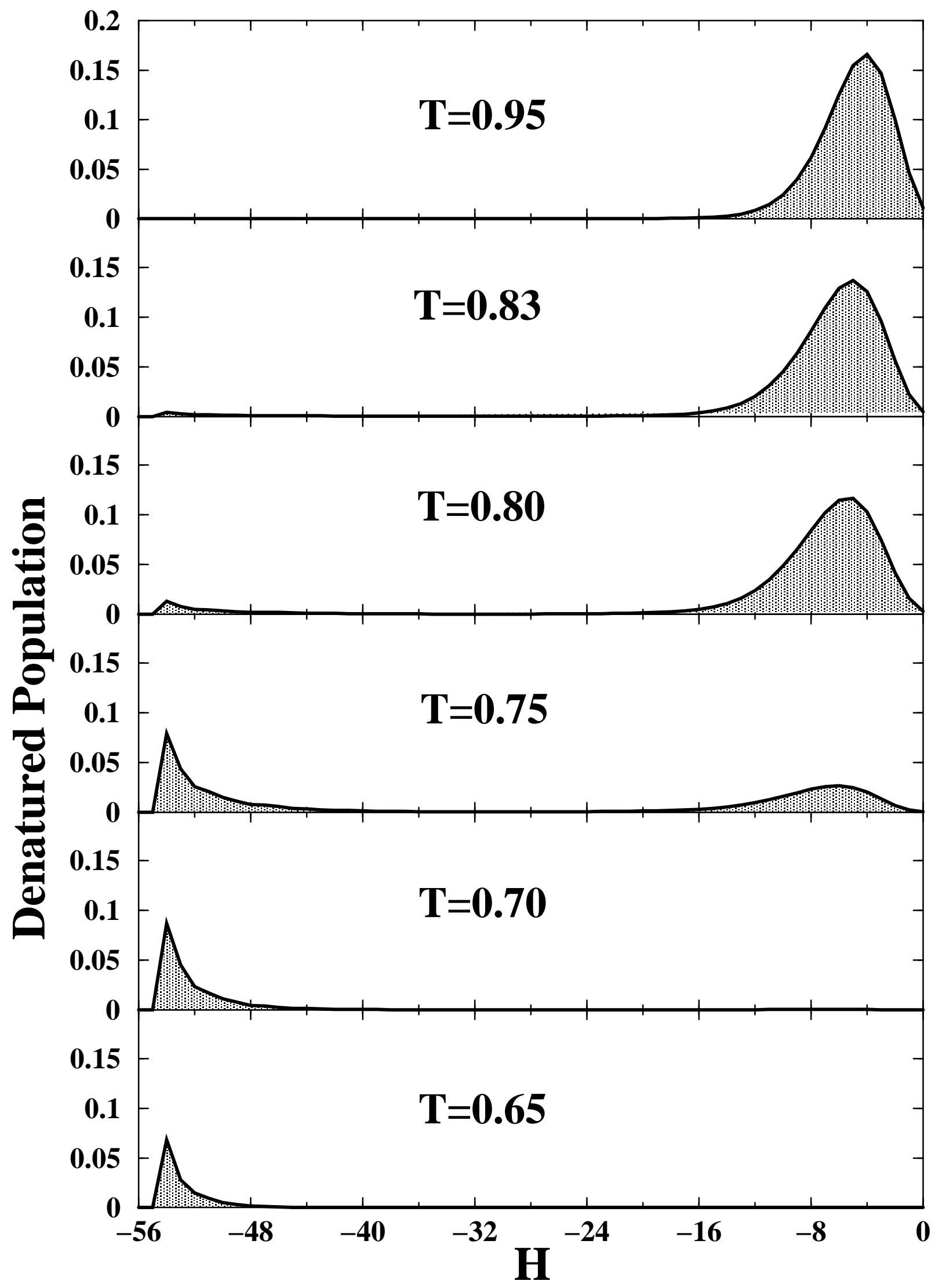



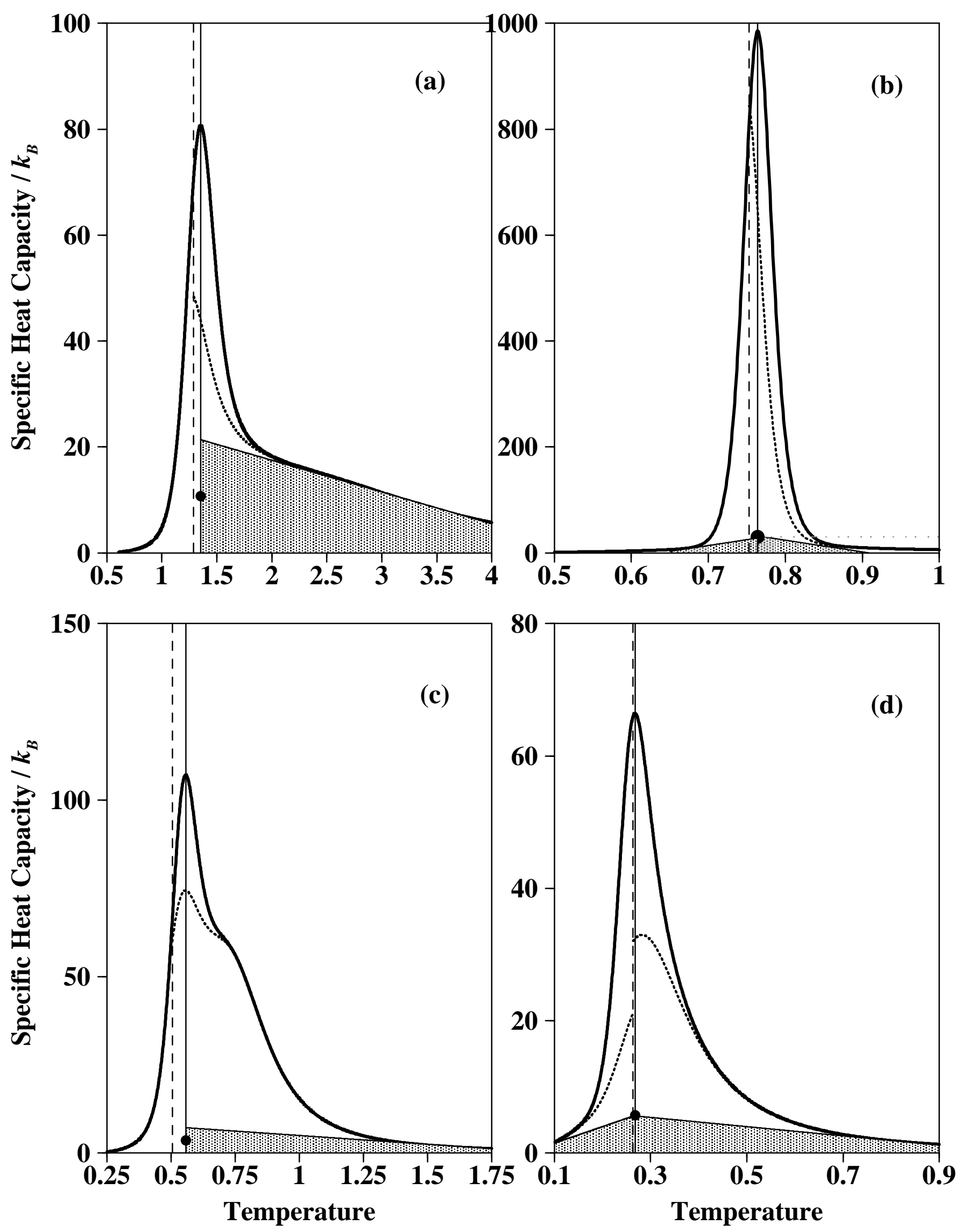

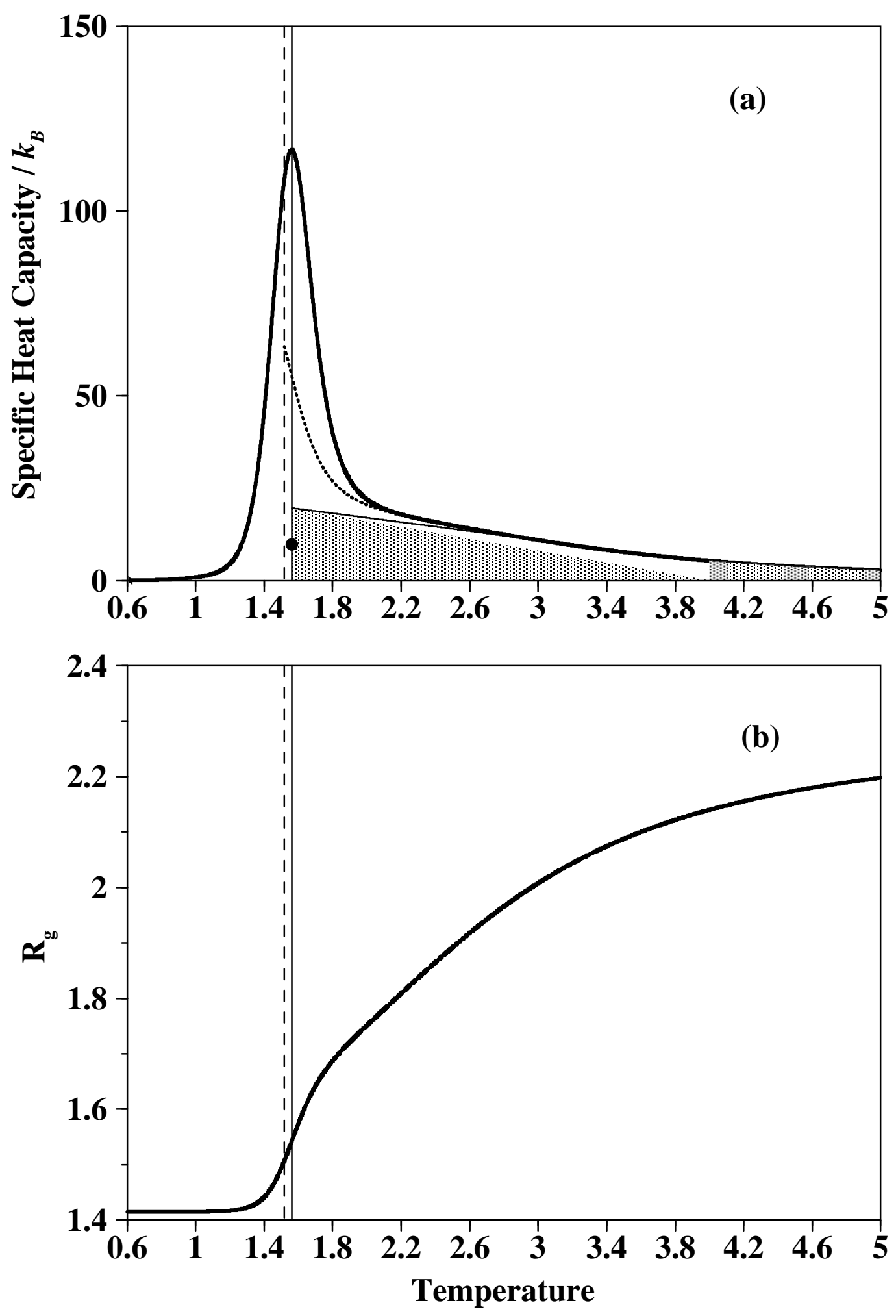

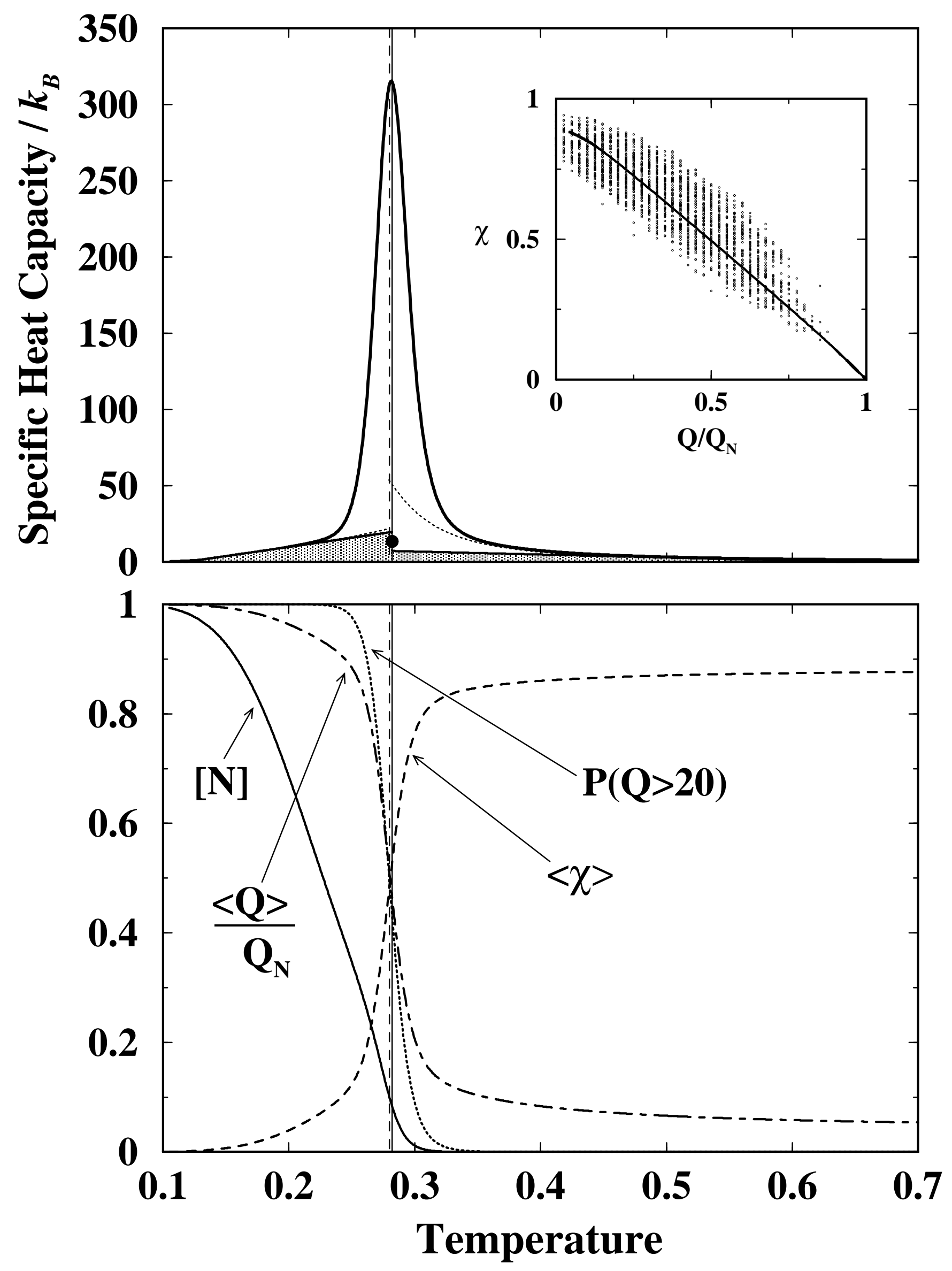

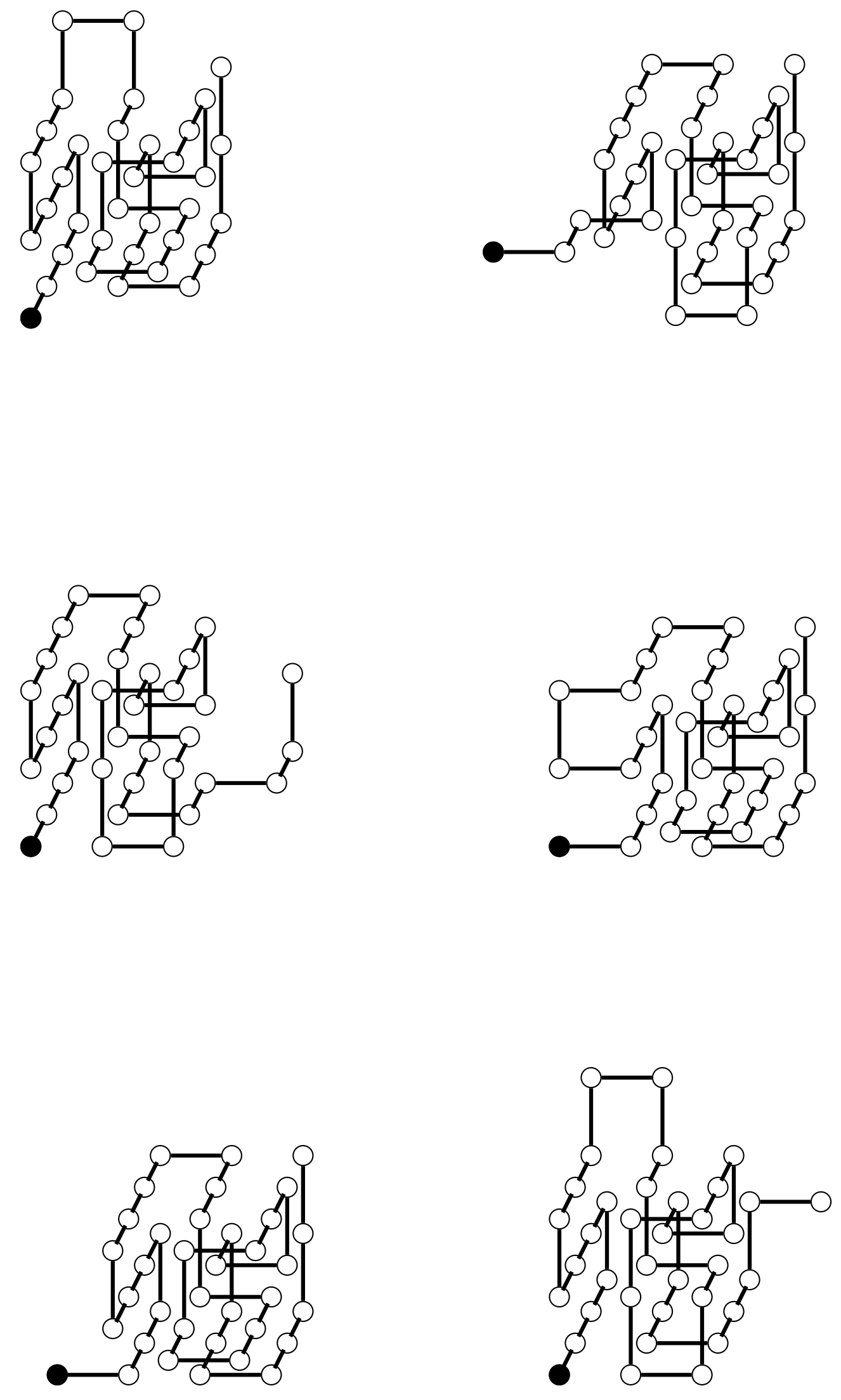

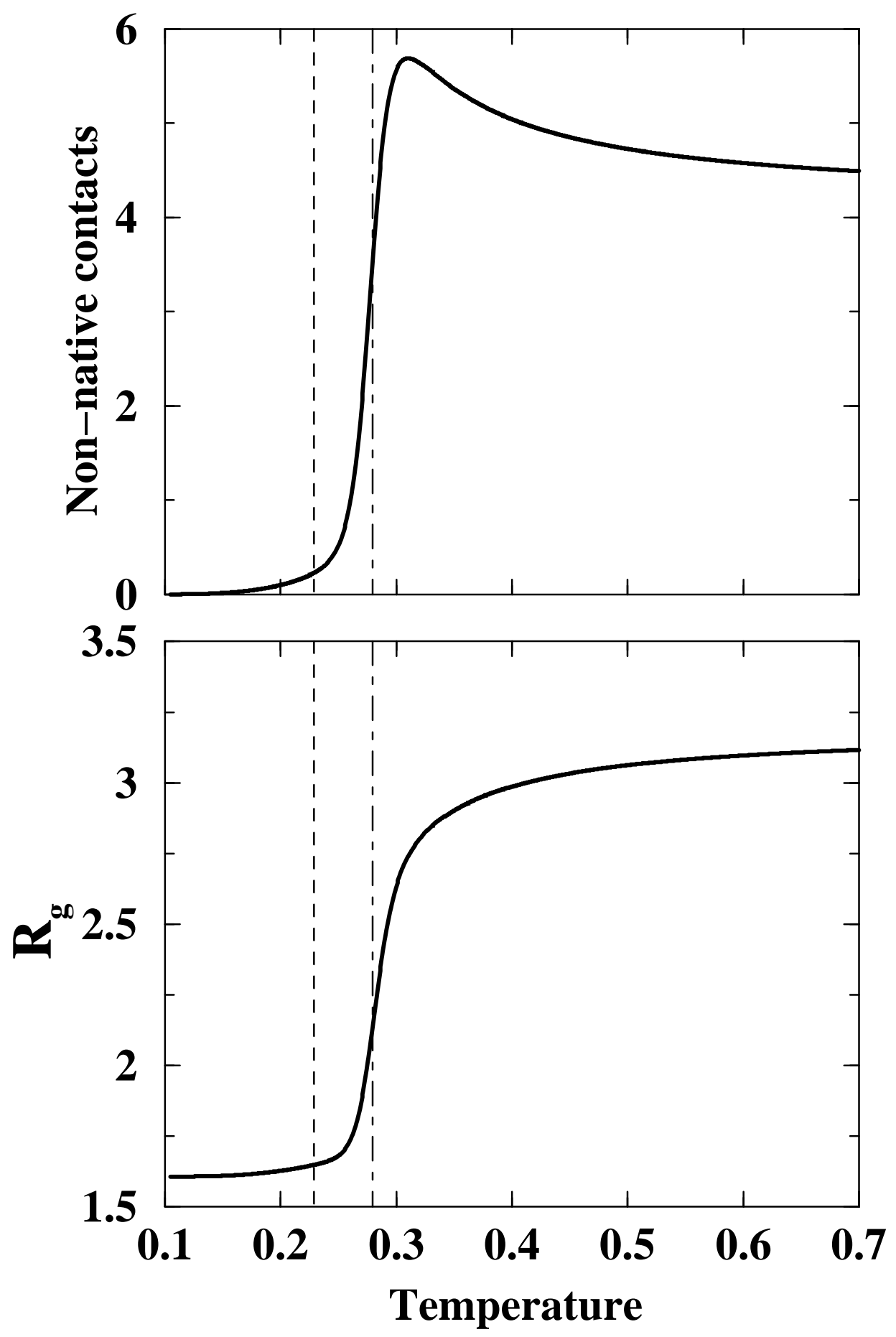